\title{
Retinitis Pigmentosa Results in Neurodegeneration Concomitant With Neuroinflammation, Extracellular Matrix Disorganization and the Upregulation of Neuroprotective Pathways in Glial Cells and Neurons
}

\section{Christina B. Bielmeier}

Universität Würzburg: Julius-Maximilians-Universitat Wurzburg

\section{Saskia Roth}

Universität Würzburg: Julius-Maximilians-Universitat Wurzburg

Sabrina I. Schmitt

Universität Regensburg: Universitat Regensburg

Stefaniya K. Boneva

Universität Freiburg: Universite de Fribourg

Anja Schlecht

Universität Freiburg: Universite de Fribourg

Mario Vallon

Universität Würzburg: Julius-Maximilians-Universitat Wurzburg

Ernst R. Tamm

Universität Regensburg: Universitat Regensburg

\section{Süleyman Ergün}

Universität Würzburg: Julius-Maximilians-Universitat Wurzburg

\section{Andreas Neueder}

Universität Ulm: Universitat Ulm

Barbara M. Braunger ( $\sim$ Barbara.Braunger@uni-wuerzburg.de.de )

Julius-Maximilian-University https://orcid.org/0000-0002-3926-6725

\section{Research}

Keywords: Retinitis Pigmentosa, VPP mouse model, In-Situ hybridization, Neurodegeneration, Neuroinflammation, Extracellular matrix disorganisation, Neuroprotective pathways

Posted Date: November 10th, 2020

DOI: https://doi.org/10.21203/rs.3.rs-101559/v1 
License: (c) (i) This work is licensed under a Creative Commons Attribution 4.0 International License. Read Full License 


\section{Abstract}

\section{Background}

Hereditary retinal degenerations like retinitis pigmentosa (RP) are amongst the leading causes of blindness in younger patients. To enable in vivo investigation of cellular and molecular mechanisms responsible for photoreceptor cell death and to allow testing of therapeutic strategies that could prevent retinal degeneration, animal models have been created. Here, we in-depth characterized the transgenic VPP mouse model, a genetic model for autosomal dominant RP.

\section{Methods}

We examined the degree of photoreceptor degeneration and studied the impact of the VPP transgeneinduced retinal degeneration on the transcriptome level of the retina using next generation RNA sequencing (RNASeq) analyses followed by weighted correlation network analysis (WGCNA). We furthermore identified cellular subpopulations responsible for some of the observed dysregulations using in situ hybridizations, immunofluorescent staining and 3D reconstruction.

\section{Results}

One month-old VPP mice showed a significantly higher number of apoptotic photoreceptor cells that resulted in a significantly thinner ONL in three months-old VPP mice, concomitant with an increase in reactivity of microglia and Müller cells. By RNASeq analysis we identified 9,256 dysregulated genes and six significantly associated gene modules in the subsequently performed WGCNA. Gene ontology enrichment showed, amongst others, dysregulation of TGF- $\beta$ regulated extracellular matrix organization, factors of the (ocular) immune system/response and apoptosis.

\section{Conclusion}

The predominant effect pointed towards induction of neuroinflammation and the upregulation of neuroprotective pathways like TGF- $\beta$, G-protein activated and VEGF signaling that were significantly associated with the VPP transgene-induced photoreceptor degeneration. Thus, modulation of these processes might represent new therapeutic options to delay the degeneration of photoreceptors in diseases like RP.

\section{Introduction}

Retinitis pigmentosa (RP) is a hereditary form of retinal degeneration that results from mutations in any one of more than 70 known susceptibility genes $(1,2)$. Quite intriguingly, RP is considered as one of the most common hereditary diseases that is associated with mutations in core splicing proteins resulting in the altered regulation of gene expression (2). Even though RP is considered a rare genetic disorder, it is still amongst the major causes of blindness in younger patients $(3,4)$ caused by the progressive loss of rod and cone photoreceptor cells, respectively (1). Photoreceptors are the light sensitive neurons of the 
retina that are responsible for visual perception (5). These cells consist of the outer and inner segments which are connected through a cilium with the cell's perikarya that are located in the outer nuclear layer (ONL) and finally build a synaptic layer in the outer plexiform layer (OPL) of the retina to signal to the inner retinal neurons $(4,5)$. Photoreceptor degeneration typically results in a thinning of the ONL concomitant with the loss of the inner and outer photoreceptor segments resulting in an impaired visual function up to blindness. Based on the genetic heterogeneity of RP, it is still a challenge to understand and more importantly to try to modify the molecular mechanisms leading to the degeneration of photoreceptors with the overall goal to delay their degeneration. Consequently, animal models mimicking photoreceptor degeneration are frequently used to gain insights into the impact of certain mutations on the degeneration of photoreceptors. Amongst these, the VPP mouse model is a well-known animal model for photoreceptor degeneration. VPP mice carry a rhodopsin transgene with five point mutations: three amino acid substitutions: Val-20 à Gly (V20G), Pro-23 à His (P23H) and Pro-27 à Leu (P27L) (VPP) and two restriction fragment length polymorphisms (6), with the $\mathrm{P} 23 \mathrm{H}$ mutation being the most prevalent mutation in US patients suffering from autosomal dominant RP (7-9). Hemizygous VPP mice harbor two to five copies of the mutant rhodopsin transgene at a single integration site in addition to the wildtype rhodopsin gene (6). Transgene expression results in slowly progressing degeneration of rod and cone photoreceptors (6). In the current study, we analyzed the morphology and apoptotic events in retinae of VPP mice. In humans, the various RP mutations of core splicing proteins result in an altered transcriptome. Therefore, we asked the question whether mutations of other genes e.g. the rhodopsin gene might also influence the retinal transcriptome with the overall aim to identify molecular key factors and signaling pathways that predominantly influence the course of photoreceptor degeneration. Thus, we studied the impact of the VPP mutation on the retinal transcriptome using RNASeq analyses, which has not been done so far. In summary, we identified more than 9,000 dysregulated genes. Performing gene correlation network analyses we identified six significantly associated modules in three-month-old VPP animals. Gene ontology enrichment analyses showed, amongst others, involvement of components of the (ocular) immune system or response, respectively, transforming growth factor $\beta$ (TGF- $\beta$ ) regulated extracellular matrix organization and apoptosis. The progressive photoreceptor loss was highlighted by e.g. a downregulation of rhodopsin mediated signaling pathway and the reactivity of (micro)glial cells. In summary, our findings show that apoptosis, neuroinflammation and the upregulation of neuroprotective pathways like TGF- $\beta$-, endothelin- and vascular endothelial growth factor (VEGF)-signaling are amongst the dominant effects following hereditary retinal degeneration in the VPP retina.

\section{Materials And Methods}

\subsection{Mice}

The mice were in a 129 SV background and kept in a $12 \mathrm{~h}$ light/dark cycle. Mice of both sexes were used for the experiments. Unrecombined mice, carrying two floxed Tgfbr2 alleles at chromosome 9 (10), thus representing functional wildtype mice, were crossbred with hemizygous VPP mice (6). Resulting offspring expressed either wildtype rhodopsin (named as control mice = VPP negative animals) or the VPP 
transgene (referred to as VPP mice), a rhodopsin mutant with point mutations at positions $\underline{\mathrm{V}} 20 \mathrm{G}, \underline{\mathrm{P}} 27 \mathrm{~L}$ and $\underline{\mathrm{P}} 23 \mathrm{H}$, in addition to wildtype rhodopsin. The VPP mutation results in a progressive retinal neurodegeneration (6). For genotyping, the following primers were used: $5^{\prime}$ agactgacatggggaggaattcccaga- $3^{\prime}$ (sense) and 5'-gagctgctcgaagtgactccgacc-3' (antisense). The thermal cycle protocol was denaturation at $94^{\circ} \mathrm{C}$ for $30 \mathrm{sec}$, annealing at $68^{\circ} \mathrm{C}$ for $45 \mathrm{sec}$ and elongation at $72{ }^{\circ} \mathrm{C}$ for $45 \mathrm{sec}$ for 35 cycles.

\subsection{Microscopy and morphometric analyses (Spider diagram)}

The enucleated eyes were fixed for $24 \mathrm{~h}$ in $2.5 \% \mathrm{PFA} / 2.5 \%$ glutaraldehyde in sodium cacodylate buffer and processed as described previously (11). $1 \mu \mathrm{m}$ thick semithin meridional sections were cut and stained after Richardson (12). The sections were analyzed on an Axio Imager Z1 microscope (Carl Zeiss, Jena, Germany) using Zeiss Zen software (Carl Zeiss, Jena, Germany). Thickness of the outer nuclear layer (ONL) was measured and the mean values were plotted as spider diagram as described previously in $(13,14)$.

\subsection{Apoptosis: TdT-mediated dUTP-biotin nick end labeling (TUNEL)}

TUNEL (DeadEnd Fluorometric TUNEL, Promega, Madison, WI, USA) was used to label apoptotic cells in one month-old animals, following our previously published protocol $(15,16)$. The sections were analyzed on an Axio Imager Z1 microscope (Carl Zeiss, Jena, Germany) using Zeiss Zen software (Carl Zeiss, Jena, Germany). TUNEL-positive cells were counted and normalized to the area of the ONL $\left[\mathrm{mm}^{2}\right]$.

\subsection{Immunofluorescence and RNA/Basescope ${ }^{\circledR}$ in situ hybridization}

Eyes were fixed for $4 \mathrm{~h}$ in $4 \%$ PFA, washed extensively in phosphate buffer (PB, $0.1 \mathrm{M}, \mathrm{pH} 7.4)$ and embedded in paraffin according to standard protocols. Paraffin sections ( $6 \mu \mathrm{m}$ thick) were deparaffinized. Glial fibrillary acidic protein (GFAP) (Agilent Dako, Santa Clara, USA), ionized calcium-binding adapter molecule 1 (IBA1) (Wako, Ōsaka, Japan) and glutamine synthetase (GS) (Millipore, Temecula, USA) immunofluorescent staining was performed as described in (13). For in situ hybridization (ACD, Newark, USA) paraffin sections were pretreated with retrieval reagent and protease according to the user manual. RNAscope ${ }^{\circledR}$ Fluorescent Multiplex Reagent Kit was used to hybridize chemokine (C-C motif) ligand 2 (CC/2) (ACD catalog number: 311791) and Endothelin 2 (Edn2) (ACD catalog number: 418221) and BaseScope $^{\mathrm{TM}}$ Detection Reagent Kit v2 - RED was used to label Vegf receptor type 2 (Vegfr2) (ACD catalog number: 860711), endothelin receptor type b (Ednrb) (ACD catalog number: 706471) and TGF- $\beta$ 
receptor type 2 (Tgfbr2) (ACD catalog number: 845871). The sections were analyzed on an Axio Imager Z1 microscope with the Apotome.2 function (Carl Zeiss, Jena, Germany) using Zeiss Zen software (Carl Zeiss, Jena, Germany). To study the co-localization of RNA/BaseScope and GFAP/GS , z-stacks (25-30 images, in total 5.5-7.0 $\mu \mathrm{m}$ thick) were analyzed, transformed into the ortho (orthogonal section) view and reconstructed as 3-D image using the Zeiss Zen software and the 3DxI rendering module with the surface function (Carl Zeiss, Jena, Germany).

\subsection{RNA isolation and quantitative real-time RT-PCR (qPCR)}

TriFast (Peqlab, Erlangen, Germany) was used to isolate total mRNA from retinal tissue and cDNA was synthesized using the iScript cDNA Synthesis Kit (Bio-Rad Laboratories, Inc., Hercules, CA, USA) following the manufacturer's instructions. QPCR analyses were performed on a CFX Realtime PCR Detection System (Bio-Rad Laboratories, Inc.) and as previously described (17). All oligonucleotides (supplementary Table 1) were designed to span exon-intron boundaries and purchased from Invitrogen (Carlsbad, California, USA). CFX Manager ${ }^{\mathrm{TM}}$ Software and Excel were used to analyse relative mRNA expression levels according to the $\Delta \Delta \mathrm{C}_{\mathrm{T}}$-method (18). To perform RNA sequencing total RNA of the retinae was purified using the RNeasy Mini Kit by Qiagen (Venlo, Netherlands).

\subsection{RNA sequencing}

Library preparation and RNAseq were performed at the service facility 'KFB - Center of Excellence for Fluorescent Bioanalytics' (Regensburg, Germany. www.kfb-regensburg.de). Library preparation and RNAseq were carried out as described in the Illumina TruSeq Stranded mRNA Sample Preparation Guide, the Illumina NextSeq 500 System Guide (Illumina, Inc., San Diego, CA, USA), and the KAPA Library Quantification Kit - Illumina/ABI Prism User Guide (Kapa Biosystems, Inc., Woburn, MA, USA). In brief, 250 ng of total RNA was used for purifying the poly-A containing mRNA molecules using poly-T oligoattached magnetic beads. Following purification, the mRNA was fragmented to an average insert size of 200-400 bases using divalent cations under elevated temperature ( $94^{\circ} \mathrm{C}$ for 4 minutes). Next, the cleaved RNA fragments were reverse transcribed into first strand cDNA using reverse transcriptase and random hexamer primers. Actinomycin D was added to improve strand specificity by preventing spurious DNAdependent synthesis. Blunt-ended second strand cDNA was synthesized using DNA Polymerase I, RNase $\mathrm{H}$ and dUTP nucleotides. The incorporation of dUTP, in place of dTTP, quenched the second strand during the later PCR amplification, because the polymerase does not incorporate past this nucleotide. The resulting CDNA fragments were adenylated at the 3 ' ends, the indexing adapters were ligated and subsequently specific cDNA libraries were created by PCR enrichment. The libraries were quantified using the KAPA SYBR FAST ABI Prism Library Quantification Kit. Equimolar amounts of each library were sequenced on a NextSeq 500 instrument controlled by the NextSeq Control Software (NCS) v2.2.0, using a 75 Cycles High Output Kit with the single index, paired-end (PE) run parameters. Image analysis and 
base calling were done by the Real Time Analysis Software (RTA) v2.4.11. The resulting .bcl files were converted into .fastq files with the CASAVA Software v1.8.2.

\subsection{Bioinformatics}

Fastq files were quality controlled with FastQC v0.11.5 (19). All files passed quality control. The reads were aligned against Ensembl Mus musculus GRCm38 version 94 using STAR aligner v2.5.3a (20). One sample (R21753) showed poor read alignments of less than $30 \%$ and was removed from further analyses. Reads were quantified using salmon v0.8.2 (21). All subsequent analyses were conducted in $R$ v3.5.1. Samples were screened for outliers using PCA and clustering analysis. One sample (R21741) was identified as an outlier and removed from further analyses. Thus, the final sample number was six control and five VPP retinae. Transcriptional dysregulation was computed using tximport v1.10.0 (22) and DESeq2 v1.22.1 (23) with genotype as the variable of interest and sex of the mice as a covariate and using ashr (24) as the fold change shrinkage estimator. The Benjamini-Hochberg procedure was used to correct for multiple comparisons ( $p$-adjusted; $p_{\text {adj }}$ ). For correlation network analysis we used the normalized and variance stabilized counts from the DESeq2 analysis. Batch correction for sex was applied with limma v3.38.3 (25) keeping genotype as the variable of interest. The normalized, transformed and batch corrected counts were used to construct a weighted gene correlation network using WGCNA v1.66 $(26,27)$. Heatmaps and k-mer analysis was carried out using ComplexHeatmap v2.3.2 (28). Visualization was carried out using cytoscape v3.7.2 (http://cytoscape.org) with the Reactome FI app v7.2.1 (29). Ontology analysis was carried out using the Enrichr website (30, 31). Scripts are available upon request.

\subsection{Statistics}

All results that are displayed in bar graphs are expressed as means \pm SEM. Data were screened for outliers using the Grubb's outlier test in graph pad prism. Comparisons between the means of two groups were made by a two-tailed Student's t-test. $p \leq 0.05$ was considered as statistically significant.

\section{Results}

\subsection{Analysis of photoreceptor degeneration in VPP mice}

To assess the degree of apoptosis in retinae of VPP mice, we visualized apoptotic cells in retinae of onemonth-old animals by TUNEL-labelling. Retinae of control animals showed only very few apoptotic cells (Figure 1A and B). In contrast VPP mice showed a considerable number of TUNEL-positive cells in the outer nuclear layer (ONL) (Figure 1A and B). Intriguingly, the wide majority of apoptotic cells was located in the central part of the retinae near the optic nerve head (ONH) (Figure 1B). When quantifying the number of the apoptotic cells on retinal sections through the $\mathrm{ONH}$ of one-month-old mice (Figure 1C) we found $16.28 \pm 3.08$ apoptotic cells per $\mathrm{mm}^{2} \mathrm{ONL}$ in retinae of control animals and $173.57 \pm 17.84$ cells 
undergoing apoptosis in VPP retinae $(p<0.001)$. To investigate, whether the apoptosis of photoreceptors affected retinal morphology, we analyzed semithin sections of the eyes of three-month-old control and VPP animals. We did not observe obvious morphological changes in retinae of control animals (Figure 1D and F). However, VPP mice showed a distinct thinning of the retina that was most pronounced in the central retina (Figure 1D and F). For quantitative analysis, we measured the thickness of the ONL layer at defined measuring points along both hemispheres of sagittal semithin-sections though the $\mathrm{ONH}$. The mean values of these individual measurements were plotted in a spider diagram (Figure 1E) and subsequent statistical analysis confirmed a significant thinning of the ONL in VPP mice compared to controls. We furthermore aimed to validate the VPP model by analyzing the mRNA expression levels by qPCR of factors like leukemia inhibitory factor (Lif), fibroblast growth factor 2 (Fgf2) and endothelin 2 $(E d n 2)$ that are well-known to be upregulated in the context of retinal degenerations $(15,32,33)$. Accordingly, we found them significantly upregulated in retinae of three-month-old VPP mice (Lif: $22.38 \pm$ 4.13, $p=0.008$; Fgf2: $13.17 \pm 1.52, p=0.005$; Edn2: $46.33 \pm 5.64, p<0.001$ ) compared to retinae of control littermates (Lif. $1.00 \pm 0.18 ;$ Fgf2: $1.00 \pm 0.14 ;$ Edn2: $1.00 \pm 0.15$ ) (Figure 1G).

\subsection{Transcriptional alterations in VPP retinae: RNAseq and weighted correlation network analysis (WGCNA)}

Subsequent, we applied next generation RNA sequencing (RNAseq) analyses to investigate the impact of VPP transgene expression and concomitant photoreceptor degeneration on the transcriptome of the retina in three-month-old VPP and control animals. Out of the total of 54,532 genes in the Ensembl annotation (Mus musculus GRCm38 v. 94) we found 30,796 genes to be expressed in the retina, of which 9,256 were dysregulated (4,636 down- and 4,620 upregulated, Figure 2A, cut off criteria: BenjaminHochberg adjusted $p$-value $\left.\left(p_{a d j}\right)<0.05\right)$. The top 30 dysregulated genes are shown in supplementary Table 2. Amongst others, genes regulating processes in neurotransmission like histidine decarboxylase (Hdc),galactosidase beta 1 like 3 (G/b1/3), which is associated with Leber's congenital amaurosis as well as serine protease 56 (Prss56) that was reported to be involved in eye development, were significantly downregulated. Genes controlling scar formation, such as fibrinogen-like 2 (Fg/2) as well as apoptosis e.g. caspase1 (Casp1) or Bcl2-interacting killer (apoptosis-inducing) (Bik), were upregulated. Furthermore, we found upregulation of quite a considerable number of genes associated with inflammatory or immune response functions such as C-X-C motif chemokine ligand 13 (CXCl13), glial fibrillary acidic protein (Gfap), T-cell receptor $\mathrm{T3}$ gamma chain (Cd3g), chemokine (C-C motif) ligand 5 (Cc/5), C-C motif chemokine ligand $2(\mathrm{Ccl} 2)$ and factors, that are associated with the complement cascade like complement component factor i (Cfi), complement factor $C 4 B$ and the Serping1 gene.

Gene ontology enrichment showed, amongst others, involvement of TGF- $\beta$ regulated extracellular matrix organization, response to cytokine stimuli and apoptosis (Table 1). Photoreceptor loss was indicated by downregulation of rhodopsin signaling pathway components (Table 1). Moreover, we performed weighted gene correlation network analysis (WGCNA) to identify genotype-specific patterns of dysregulation, upstream regulators and involved signaling pathways. WGCNA clusters co-regulated genes into modules 
based on their similarity of expression. Since this approach does not rely on the traditional dysregulation analysis and the problem of correction for multiple comparisons, more subtle changes and patterns can be identified. In addition, biological key players, e.g. regulatory proteins driving a certain pathway, for a given module can be found by the intra-module analysis. The topology overlay matrix, which represents the co-regulation of expression, for VPP and control animals, as well as the identified modules (clusters of co-regulated genes as shown by their colors in Figure 2B) and their correlation of expression with the genotype, are illustrated in Figure 2B. The analysis identified six significantly associated modules (three positively correlated with the genotype, i.e. higher expressed in the VPP animals (Pos1, 2, 3) and three negatively correlated, i.e. lower expressed in the VPP animals (Neg1, 2, 3) (Figure 2D and E and supplementary Figure 1A - D). The Pos1 module was significantly enriched for genes involved in cellular transport and differentiation (Figure $2 \mathrm{D}$ and Table 2). In the Pos2 module, we found significant clustering of genes encoding for necroptosis, protein transport, organelle organization, cellular homeostasis, and the ribosome (supplementary Figure 1A and Table 2). The Pos3 module (supplementary Figure 1B and Table 2) showed enrichment for bone morphogenetic protein (BMP) signaling pathway components. In the Neg1 module we observed significant clustering of genes regulating cellular component organization and protein kinase activity (Figure $2 \mathrm{E}$ and Table 2). The Neg2 module (supplementary Figure $1 \mathrm{C}$ and Table 2) was enriched for genes involved in nucleotide homeostasis and the Neg3 module showed significant enrichment for genes encoding for ribosomal proteins and protein transport (supplementary Figure 1D and Table 2).

\subsection{The glial response to photoreceptor degeneration in VPP mice}

In addition to the quantitative information from the RNAseq data and to further validate its results, we performed mRNA in situ hybridization and/or immunofluorescence staining to analyze the cell types expressing transcripts of interest. As we observed a significant upregulation of several genes regulating glial reactivity (e.g. glial fibrillary acidic protein (Gfap), serine protease inhibitor A3N (Serpina3n), lipocalin 2 (Lcn2), we performed immunofluorescence labelling of retinal sections for GFAP. GFAP is a major intermediate filament particularly expressed in astrocytes (34), which is upregulated in response to retinal trauma in astrocytes and Müller cells $(34,35)$. Immunofluorescence staining against GFAP in threemonth-old control mice showed the characteristic staining pattern for astrocytes on top of the retina for both groups (Figure $3 \mathrm{~A}$ and B). Consistent with the observed upregulation of Gfap in VPP retinae as determined by RNAseq analysis (17.05-fold, $\left.p_{a d}=3.87 \cdot 10^{-78}\right)$, immunofluorescence staining showed an increased GFAP signal intensity, indicating an enhanced protein expression, on top of the retina and an additional stripe-like staining pattern stretching through the retina, which represents the characteristic morphological appearance of reactive Müller cells. Unlike apoptosis and ONL thinning, we could not detect a difference in GFAP reactivity between the central and the peripheral parts of the retina (Figure $3 \mathrm{~A}$ and B). To further validate the RNAseq data, we performed qPCR analyses on retinal samples to determine the relative Gfap expression levels, and also the expression levels of the 
microglia/macrophage marker ionized calcium-binding adapter molecule 1 (Iba1) and the chemokine (C-C motif) ligand 2 ( $C \mathrm{Cl} 2)$ which is reported to stimulate the migration and reactivity of microglia cells $(36,37)$. QPCR analyses confirmed that these mRNA expression levels were significantly upregulated in VPP retinae (Gfap: $8.89 \pm 0.63, p<0.001 ;$ Iba1: $6.56 \pm 1.03, p=0.015 ; C c / 2: 68.74 \pm 5.11, p<0.001$ ) compared to control retinae (Gfap: $1.00 \pm 0.19 ; \mathrm{Iba1}: 1.00 \pm 0.11 ; \mathrm{Ccl} 2: 1.00 \pm 0.11$ ) (Figure 3C). In accordance, our RNAseq data showed comparable increase of $I b a 1$ (5.51-fold, $p_{a d j}=1.12 \cdot 10^{-23}$ ) and $C c / 2$ mRNA (67.51-fold increase, $p_{a d j}=8.05 \cdot 10^{-14}$; Figure 3F). Furthermore, we used an anti-IBA1 labeling to visualize myeloid cells e.g. microglia and recruited macrophages, in the retinae of control and VPP animals (Figure 3D). In controls, we observed ramified IBA1-positive cells in their typical localization on top of the retina and the inner (IPL) and outer plexiform layers (OPL). In contrast, in VPP retinae, IBA1positive cells changed their shape from ramified microglia towards amoeboid, reactive microglia in particular in the OPL and thus in very close association with the degenerating photoreceptors. Moreover, we observed an accumulation of amoeboid-shaped, IBA1-positive cells in the sub-neuroretinal space in close proximity to the retinal pigment epithelium (RPE) (Figure 3D). Taken together, these results showed a pronounced reactivity of macro- and microglia cells in response to photoreceptor degeneration. To further study the origin of the significantly elevated $C c / 2$ expression in VPP retinae and to supplement the quantitative information from the RNAseq (Figure 3F) and APCR data (Figure 3C), we performed Ccl2 mRNA in situ hybridization on retinal sections combined with immunofluorescence co-labeling of glial cells. Immunofluorescence staining for glutamine synthetase (GS) staining was used to label Müller cells (38) and for GFAP to detect astrocytes and reactive GFAP-positive Müller cells. In control retinae, we observed a rather low number of $C c / 2$ punctae in the inner nuclear layer (INL), the ONL and a few signals in the retinal ganglion cell layer (GCL) (Figure 3E and supplementary Figure $4 \mathrm{~A}$ and $\mathrm{B}$ ). The number of $C c / 2$ punctae was markedly increased in the INL and ONL of the VPP retinae (Figure 3E). When using Cc/2/GFAP/GS co-labeling we observed sparse overlap of Cc/2 in GFAP-positive astrocytes (Figure 3G and supplementary Figure 4A) and more frequent overlap in GS-positive resting and in GFAP-/GS-positive reactive Müller cells (Figure 3H and supplementary Figure 4B). However, we also detected $\mathrm{Cc} / 2 \mathrm{mRNA}$ expression in cells other than Müller glia and astrocytes in the neuronal layers of the retina (GCL, INL and $\mathrm{ONL}$ ) pointing towards additional expression in retinal neurons (Figure 3E).

\subsection{Dysregulation of potentially neuroprotective pathways in VPP retinae: VEGF-, TGF- $\beta$ - and G-protein mediated signaling}

As a follow up to our previously published studies $(13,15,16)$ on the neuroprotective properties of signaling pathways such as the transforming growth factor (TGF) - $\beta$ signaling, G-protein activated signaling and vascular endothelial growth factor (VEGF) mediated signaling, we investigated their potential regulation in the VPP model. Quite intriguingly, our RNAseq data analysis (supplementary Table 2) showed a significant upregulation of genes encoding for members of the G-protein activated signaling family. Here we particularly focused on the endothelin family, as our group and others recently showed 
that endothelin 2 (Edn2) and endothelin receptor b (Ednrb) are upregulated following photoreceptor damage $(15,32,33,39)$. In accordance the RNAseq data (supplementary Table 2 ) of the VPP retinae showed a significant increase in Ednrb (1.28-fold, $\left.p_{a d j}=0.0074\right)$ and Edn2 (22.12-fold, $p_{a d j}=8.68 \cdot 10^{-87}$ ) expression. We furthermore observed an upregulation of factors involved in TGF- $\beta$ signaling (e.g. Tgf- $\beta$ receptor type 1 (Tgfbr1): 1.15-fold, $p_{\text {adj }}=0.013 ;$ Tgfbr2: 2.23 -fold, $p_{\text {adj }}=2.18 \cdot 10^{-23} ;$ Tgf- $\beta 1: 2.24, p_{a d j}=$ $1.24 \cdot 10^{-12} ;$ Tgf- $\left.\beta 2: 1.51-\mathrm{fold}, p_{\text {adj }}=2.20 \cdot 10^{-20}\right)$. Of note, $\operatorname{Tgf}-\beta 2$ was additionally identified as one of the hub gene in the Pos1 module. Vascular endothelial growth factor (VEGF) receptor signaling pathway was identified in the gene ontology enrichment analysis of the significantly upregulated genes and the RNAseq data (supplementary Table 2) showed an increased expression of Vegfr1 (Flt1): 1.25, $p_{\text {adj }}=$ 4.75·10-7, Vegfr2 (Kdr): 2.14, $p_{a d j}=1.40 \cdot 10^{-41} ;$ Vegfb: $1.20, p_{a d j}=0.00013 ;$ and Vegfc: $1.46, p_{a d j}=9.31 \cdot 10^{-}$ 5 .

Thus, we aimed to investigate the impact of these signaling pathways (TGF- $\beta-$ - G-protein activated- and VEGF- signaling) on the VPP model in detail. Unsupervised hierarchical clustering of the samples that we generated on basis of the Reactome pathway database (29) for the VEGF (Figure 4A), TGF- $\beta$ (Figure 4B) and G-protein mediated signaling pathways (Figure $4 C$ ) demonstrated a perfect separation of the genotypes highlighting the dysregulation of these pathways in the VPP animals. Furthermore, k-mer analysis (three k-mer groups indicated by numbers on the left side of each heatmap) showed clusters of tightly co-regulated genes. We highlighted some genes of particular interest (e.g. which are known to be involved in neuroprotective or immune modulating processes or to be involved in regulatory functions) in each pathway on the right side of each heatmap. The heatmaps including the full labelling are shown in supplementary Figure 2. To further analyze sub-groups of dysregulated pathways, we transformed the Reactome pathways into functional interaction networks (Figure $4 \mathrm{D}, \mathrm{E}$ and $\mathrm{F}$ ). We colored the genes of each network according to their dysregulation: white indicates no significant regulation, red genes were up- and blue genes were significantly down-regulated, respectively. The size of each node corresponds to the log2-fold change of regulation. The fully labeled networks are shown in supplementary Figure 3 . This analysis identified distinct sub-clusters of dysregulated genes, e.g. endothelin 2 (Edn2) and endothelin receptor $b(E d n r b)$ in the G-protein activated signaling pathway network (Figure 4E and supplementary Figure $3 \mathrm{C}$ ) or Tgfbr2 in the TGF- $\beta$ family signaling network (Figure 4D and supplementary Figure 3B) and Vegfr2/kinase insert domain receptor (Kdr) in the VEGF signaling network (Figure 4F and supplementary Figure 3A).

Next, we used RNA/BaseScope ${ }^{\circledR}$ in situ hybridization to supplement the quantitative information from the RNAseq/qPCR data and to identify specific cell types expressing transcripts of interest. Using a specific probe against $E d n 2$ we detected Edn2 most prominently in the ONL in the control retina. However, we also observed distinct Edn2 signals in the INL and some rather sparse signals in the GCL (Figure 5A). VPP retinae showed significantly higher $E d n 2$ expression in the RNAseq data (22.12-fold, $p_{a d j}=8.68 \cdot 10^{-87}$; Figure 5B) (supplementary Table 2)/qPCR analyses (Figure 1G) and accordingly showed a marked increase of Edn2 signals in particular in the degenerating ONL and in the INL, but the signals in the GCL remained sparse (Figure 5A). When performing GFAP/GS co-labelling, we observed some Edn2 signals in 
GFAP-positive astrocytes (Figure 5C and supplementary Figure 4C) and in GS-positive resting and GFAP/GS-positive reactive Müller cells, respectively (Figure 5 D and supplementary Figure 4D). However, we observed the majority of the Edn2 signals in the neurons of the INL and ONL (Figure 5A). Ednrb mRNA in situ hybridization showed specific signals in the INL and ONL and some defined signals in the GCL in control retinae (Figure 5E). We furthermore observed some Ednrb expression in GFAP-positive astrocytes (Figure 5G and supplementary Figure 4E) and in GS-positive resting Müller cells (Figure $5 \mathrm{H}$ and supplementary Figure 4F). In VPP retinae, Ednrb was significantly upregulated (1,29-fold, $p_{a d j}=0.007$; Figure 5F) in the RNAseq data (supplementary Table 2). In the in situ hybridization, we detected pronounced Ednrb signals in the INL and ONL that overlapped to some extent with GFAP-/GS-positive reactive Müller cells (Figure $5 \mathrm{E}$ and $\mathrm{H}$ and supplementary Figure 4F).

Tgfbr2 was significantly upregulated (2.23-fold, $p_{a d j}=2.18 \cdot 10^{-23}$; Figure 6B) in VPP retinae in the RNAseq data (supplementary Table 2). To further supplement the quantitative information and potentially identify cell types in which it is upregulated, we performed Tgfbr2 in situ hybridization. Control retinae showed distinct signals in the INL and ONL and some scattered punctae in the GCL (Figure 6A and supplementary Figure $4 \mathrm{G}$ and $\mathrm{H}$ ). In VPP retinae, the number of Tgfbr2 punctae increased in the INL and ONL.

Immunofluorescence co-labelling confirmed its expression in only some isolated GFAP-positive astrocytes (Figure 6C and supplementary Figure 4G) and its association with resting, GS-positive and reactive GFAP-/GS-positive Müller cells, respectively (Figure 6D and supplementary Figure 4H). Yet, we also observed Tgfbr2in situ hybridization in the neuronal cell layers of the retina, in particular in the INL and ONL, that did not overlap with GFAP-/GS-positive Müller cells, indicating its additional expression in neuronal cells (Figure 6A).

Vegfr2 mRNA in situ hybridization in control retinae showed numerous signals in the INL that partly overlapped with GS-positive resting Müller cells (Figure 6E and supplementary Figure 4J). Moreover, we detected Vegfr2 mRNA signals in the ONL and isolated signals in the GCL that overlapped to some extent with GFAP-positive astrocytes (Figure 6G and and supplementary Figure 4I). Our RNAseq analysis showed Vegfr2 to be significantly upregulated in VPP retinae $\left(2.00-\mathrm{fold}, p_{a d j}=1.40 \cdot 10^{-41}\right.$; Figure 6F; supplementary Table 2) and accordingly Vegfr2 in situ hybridization showed an increased in expression in the INL and ONL (Figure 6E). Co-labelling showed its association to and expression in GFAP-/GSpositive reactive Müller cells (Figure 6H and supplementary Figure 4J). Moreover, we detected Vegfr2 signals in the neuronal layers of the retina, again in particular in the INL and ONL that did not overlap with GFAP-/GS-positive Müller cells, indicating its additional expression in neuronal cells (Figure 6E).

\section{Discussion}

The present data confirm that the VPP model displays the major phenotypic characteristics of the human disease retinitis pigmentosa. Briefly, we demonstrate in comprehensive transcriptome-wide analyses of retinae from three-month-old VPP mice (1) an extensive dysregulation of genes encoding for apoptosis, processes in scar formation, and components of the (ocular) immune system or response, respectively, 
(2) a strong genotype-dependent clustering of genes regulating the VEGF, TGF- $\beta$ and G-protein activated signaling pathway, (3) the expression of regulatory genes in neurons, resting and reactive glia cells, and (4) a dysregulation of extracellular matrix organization, apoptosis and response to cytokine stimuli in WGCNA analyses.

\section{The transcriptional response to photoreceptor degeneration leads to increased expression of genes regulating inflammatory or immune response functions}

Neuroinflammation is a common hallmark of the pathogenesis of neurodegenerative diseases like Alzheimer's, Parkinson's, multiple sclerosis or retinal degenerations $(40,41)$. Following a neurotoxic event, neuronal stress signals mediate reactivity of microglial cells leading to their proliferation, migration and the secretion of specific cytokines and chemokines that can exert neurotoxic or neuroprotective effects $(41,42)$. Sustained reactivity of microglia promotes chronic inflammation and may cause irreversible neuronal cell death $(41,43,44)$. Thus, the accumulation of reactive IBA1-positive cells in the OPL and in the sub-neuroretinal space in VPP retinae strongly indicates an ongoing neuroinflammatory process. Moreover, in the top 30 dysregulated genes, we found a considerable number of genes that are associated with inflammatory or immune response functions, respectively. Gene ontology enrichment analyses also pointed towards an upregulation of the cellular response to cytokine stimuli, again indicating an ongoing neuroinflammation. These findings are in accordance with previously published data, which describe upregulation of factors like Lif, Ccl2 (Mcp-1), interleukin-1 (II-1), complement component $1 q(\mathrm{C} 1 q)$ and complement factor $\mathrm{H}(\mathrm{CFH})$ in retinae of genetic mouse models of $\mathrm{RP}(32,45$ 49). As we and others $(36,37)$ have shown $C c / 2$ is expressed in Müller cells and photoreceptors in the healthy retina and upon retinal damage contributing to the recruitment of microglia/infiltrative macrophages $(37,50)$. However, conflicting data exist, concerning the exact role of $\mathrm{Ccl} 2$ in the context of neurodegeneration. Recently, Joly and colleagues showed that the retinal morphology of double mutant mice expressing the VPP transgene on a null background for $\mathrm{Ccl} 2\left(\mathrm{VPP} ; \mathrm{Ccl}^{-1-}\right)$ did not differ to that of transgene VPP mice (32). In contrast, Rutar and colleagues demonstrated that siRNA-mediated knock down of $C c / 2$ resulted in a significantly lower number of apoptotic photoreceptors in rats after lightinduced photoreceptor degeneration and $(37,50)$. Based on our data, it is very likely that in VPP retinae the elevated $\mathrm{Ccl} 2$ expression in Müller cells and in the ONL contribute to the attraction/migration and reactivity of microglial cells in this particular region. In our comprehensive analyses, we furthermore detected a considerable number of genes encoding for components of the complement system that is part of the innate immune system. Various complement factors have been reported to be upregulated in retinae of human patients suffering from RP or in retinae of mice following genetically or light-induced photoreceptor degeneration in mice (47-49, 51-54). Activation of the complement system promotes microglia/infiltrative macrophages migration and eventually complement activated lysis $(48,51)$. Still, conflicting data exist regarding the exact role of complement system activation and its impact on photoreceptor degeneration. Mice with a deficiency in complement factor $D$ are protected from light- 
induced photoreceptor degeneration (47), indicating a detrimental role for photoreceptor survival, but the deficiency in complement component 3 (C3) or complement receptor 3 (CR3) in a genetic mouse model of photoreceptor degeneration increases microglia-mediated neurotoxicity to photoreceptors (54). Thus, the detailed function of the complement system and its specific role in microglia and Müller cells and its contribution to photoreceptor degeneration has yet to be elucidated. Nevertheless, based on our transcriptome-wide data, we conclude that the significant upregulation of genes that are associated with inflammatory or immune response functions leads to neuroinflammation in VPP retinae potentially contributing to the degeneration of photoreceptors.

\section{The transcriptional response to photoreceptor degeneration leads to the upregulation of neuroprotective factors and pathways}

We additionally analyzed the impact of the VPP model on neuroprotective pathways like TGF- $\beta$, G-protein and VEGF signaling (55). Recently, our group and others showed that in response to retinal injury, endothelin 2 (Edn2) is expressed by photoreceptors concomitant with an elevated expression of endothelin receptor $b$ (Ednrb) and Gfap, the latter indicating the reactivity of Müller cells, and an increased expression of Lif and Fgf2 $(14,15,32,33,39)$. Our RNAseq data, in situ hybridizations,

immunofluorescence staining and qPCR analyses confirmed this observation for the VPP model of retinal degeneration. It is of interest to note that our co-labelling experiments showed expression not only of Ednrb but also of Tgfbr2 and Vegfr2 in resting and reactive Müller cells, clearly indicating the close interplay of neuronal and glial cells. Furthermore, we previously showed that Edn1, Edn2, Ednra and Ednrb were significantly upregulated following induced ocular traumata (39). Yet, in VPP retinae only Edn2 and $E d n r b$, were upregulated. These inconsistent results might well be explained by the different activation patterns of signaling pathways depending on the actual cause of cell death, i.e. light induced versus genetically induced cell death (45).

\section{The transcriptional response to photoreceptor degeneration leads to upregulation of pro-apoptotic factors and extracellular matrix organization}

Our transcriptomic analyses also showed an upregulation of factors that are associated with apoptosis (Casp1, Bik) and scar formation such as fibrinogen like 2 (Fgl2) and Tgf- $\beta 1$. A well-described characteristic of TGF- $\beta$ signaling is its contribution to wound healing, tissue fibrosis and scar formation $(56,57)$. Accordingly, the TGF- $\beta$ regulated organization of extracellular matrix was a major hit in our gene ontology analyses and might well be the result of a healing response following photoreceptor degeneration in VPP retinae. We furthermore identified an upregulation of the isoform Tgf- $\beta 2$ as one of the central hub genes in the WGCNA module Pos1. Gene ontology analysis of the Pos1 module showed 
enrichment for genes involved in cell differentiation. TGF- $\beta$ signaling modulates manifold processes e.g. the regulation of early development, cell-cycle control and cell differentiation (58-60). Moreover, we recently showed that the deletion of TGF- $\beta$ signaling results in the development of retinal microaneurysms and choroidal neovascularization $(13,17)$, clearly emphasizing its potential to regulate angiogenic processes. We furthermore showed that TGF- $\beta$ signaling protects retinal neurons from programmed cell death during retinal development (16), thus highlighting its neuroprotective properties (61).

The observed gliosis of astrocytes and Müller cells as indicated e.g. by elevated Gfap expression levels and the characteristic stripe-like staining pattern of GFAP in retinal sections, is a typical reaction of neuronal tissue to various neurotoxic insults $(35,62)$ and eventually results in a glial scar. The identified dysregulation of genes involved in neuroinflammation, neuroprotection, apoptosis, scar formation and wound healing and the corresponding WGCNA data are not only of interest for researchers working on retina but might well be of interest for scientists working with other neuronal tissues.

\section{Conclusion}

The parallel expression of VPP mutant and wildtype rhodopsin (6) results in a significant increase in apoptosis and thinning of the ONL to half of its thickness in retinae of three-month-old VPP animals. Intriguingly, in our transcriptome-wide analyses, we found more than 9,000 dysregulated genes in retinae of three-month-old VPP mice. The predominant changes in gene expression point towards induction of apoptosis, scar formation, neuroinflammation, and the upregulation of neuroprotective pathways like TGF- $\beta$, G-protein activated (e.g. endothelin) and VEGF signaling in VPP retinae. Using in situ hybridizations combined with cell-type specific markers we could show that regulatory factors such as Ccl2, Edn2, Tgfbr2, Ednrb and Vegfr2 were also expressed in glial cells in addition to neurons. Thus, the modulation of these processes in general or e.g. in glial cells are promising targets for the development of new therapeutic options to delay the degeneration of photoreceptors in diseases like RP.

\section{Abbreviations}




\begin{tabular}{ll}
\hline Bik & Bcl2-interacting killer \\
\hline BMP & Bone morphogenetic protein \\
\hline C1q & Complement component 1q \\
\hline C3 & Complement component 3 \\
\hline Casp1 & Caspase 1 \\
\hline Ccl2 & Chemokine (C-C motif) ligand 2 \\
\hline Ccl5 & Chemokine (C-C motif) ligand 5 \\
\hline Cd3g & T-cell receptor T3 gamma chain \\
\hline Cfh & Complement factor H \\
\hline Cfi & Complement component factor i \\
\hline Cr3 & Complement receptor 3 \\
\hline Cxcl13 & C-X-C motif chemokine ligand 13 \\
\hline Edn1 & Endothelin 1 \\
\hline Edn2 & Endothelin 2 \\
\hline Ednra & Endothelin receptor type a \\
\hline Ednrb & Endothelin receptor type b \\
\hline Fgf2 & Fibroblast growth factor 2 \\
\hline Fgl2 & Fibrinogen-like 2 \\
\hline GCL & Ganglion cell layer \\
\hline Gfap & Glial fibrillary acidic protein \\
\hline Glb1l3 & Galactosidase beta 1 like 3 \\
\hline Gnb2l1 & Guanine nucleotide binding protein subunit beta2 like 1 \\
\hline GS & Glutamine synthetase \\
\hline Hdc & Histidine decarboxylase \\
\hline Iba1 & Ionized calcium-binding adapter molecule 1 \\
\hline INL & Interleukin-1 \\
\hline Inner nuclear layer \\
\hline Inner plexiform layer \\
\hline Kinase insert domain receptor \\
\hline If
\end{tabular}




\begin{tabular}{ll} 
Lif & Leukemia inhibitory factor \\
\hline Lcn2 & Lipocalin 2 \\
\hline ONH & Optical nerve head \\
\hline ONL & Outer nuclear layer \\
\hline OPL & Outer plexiform layer \\
\hline Prss56 & Serine protease 56 \\
\hline RNAseq & Next generation RNA sequencing \\
\hline RP & Retinitis pigmentosa \\
\hline RPE & Retinal pigment epithelium \\
\hline Serpina3n & Serine protease inhibitor A3N \\
\hline TGF & Transforming growth factor \\
\hline Tgfbr1 & Transforming growth factor - ßreceptor type 1 \\
\hline Tgfbr2 & Transforming growth factor $-\beta$ receptor type 2 \\
\hline Ubc & Ubiquitin C \\
\hline VEGF & Vascular endothelial growth factor \\
\hline Vegfr1 & Vascular endothelial growth factor receptor type 1 \\
\hline Vegfr2 & Vascular endothelial growth factor receptor type 2 \\
\hline WGCNA & Weighted correlation network analysis \\
\hline
\end{tabular}

\title{
7. Declarations
}

\section{Ethics approval and consent to participate}

All procedures conformed to the Uniform Requirements for manuscripts submitted to biomedical journals, the tenets of the National Institutes of Health Guidelines on the Care and Use of Animals in Research, the EU Directive 2010/63/E, and were approved by the Government of Bavaria, Regierung von Unterfranken, Würzburg, Germany (AZ 55.2-2532-2-1254).

\section{Consent for publication}

\author{
Not applicable
}

\section{Availability of supporting data}


The raw data files of the RNAseq data (suppl. table 2 and 3) are available from the authors upon request.

\section{Competing interests}

The authors declare that they have no competing interests.

\section{Funding}

This work was supported by PRO RETINA Deutschland e.V. (S.I.S., B.M.B.), the Jackstädt Foundation (B.M.B.), DFG grant BR 4957/3-1 (B.M.B.) and the Helmut Ecker Foundation (B.M.B, S.R.). The funders had no role in study design, data collection and analysis, decision to publish, or preparation of the manuscript.

\section{Authors` contributions}

CBB: Roles: Data curation, Formal analysis, Investigation, Methodology, Writing - original draft, Writing review \& editing

Affiliation: Institute of Anatomy and Cell Biology, Julius-Maximilians-University Wuerzburg, Wuerzburg, Germany

SR: Roles: Data curation, Formal analysis, Investigation, Methodology, Writing - review \& editing Affiliation: Institute of Anatomy and Cell Biology, Julius-Maximilians-University Wuerzburg, Wuerzburg, Germany

SIS: Roles: Data curation, Formal analysis, Investigation, Methodology, Writing - review \& editing Affiliation: Institute of Human Anatomy and Embryology, University of Regensburg, Regensburg, Germany

SKB: Roles: Data curation, Formal analysis, Investigation, Methodology, Writing - review \& editing Affiliation: Eye Center, Medical Center, Faculty of Medicine, University of Freiburg, Freiburg, Germany AS: Roles: Data curation, Formal analysis, Investigation, Methodology, Writing - review \& editing Affiliation: Eye Center, Medical Center, Faculty of Medicine, University of Freiburg, Freiburg, Germany MV: Roles: Data curation, Validation, Methodology, Writing - review \& editing Affiliation: Institute of Anatomy and Cell Biology, Julius-Maximilians-University Wuerzburg, Wuerzburg, Germany 
ERT: Roles: Resources, Supervision, Writing - review \& editing

Affiliation: Institute of Human Anatomy and Embryology, University of Regensburg, Regensburg, Germany

SE: Roles: Resources, Supervision, Writing - review \& editing

Affiliation: Institute of Anatomy and Cell Biology, Julius-Maximilians-University Wuerzburg, Wuerzburg, Germany

AN: Roles: Conceptualization, Data curation, Formal analysis, Investigation, Methodology, Software, Writing - original draft, Writing - review \& editing

Affiliation: Department of Neurology, University of Ulm, Ulm, Germany

BMB: Roles: Conceptualization, Project Administration, Supervision, Data curation, Resources, Formal analysis, Investigation, Methodology, Funding acquisition, Writing - original draft, Writing - review \& editing

Affiliation: Institute of Anatomy and Cell Biology, Julius-Maximilians-University Wuerzburg, Wuerzburg, Germany

\section{Acknowledgments}

The authors wish to thank Prof. Esther Asan for her helpful suggestions. We thank Alla Ganscher, Elke Stauber, Angelika Pach, Silvia Babl and Margit Schimmel for the great technical assistance and Christoph Möhle and Thomas Stempfl of the Kompetenzzentrum für Bioanalytik, University of Regensburg for the RNA sequencing analysis.

\section{References}

1. Hartong DT, Berson EL, Dryja TP. Retinitis pigmentosa. Lancet. 2006;368(9549):1795-809.

2. Ruzickova S, Stanek D. Mutations in spliceosomal proteins and retina degeneration. RNA Biol. 2017;14(5):544-52.

3. Buch H, Vinding T, La Cour M, Appleyard M, Jensen GB, Nielsen NV. Prevalence and causes of visual impairment and blindness among 9980 Scandinavian adults: the Copenhagen City Eye Study. Ophthalmology. 2004;111(1):53-61.

4. Farrar GJ, Kenna PF, Humphries P. On the genetics of retinitis pigmentosa and on mutationindependent approaches to therapeutic intervention. The EMBO journal. 2002;21(5):857-64.

5. Kolb H. Simple Anatomy of the Retina. In: Kolb H, Fernandez E, Nelson R, editors. Webvision: The Organization of the Retina and Visual System. Salt Lake City (UT) 1995. 
6. Naash MI, Hollyfield JG, al-Ubaidi MR, Baehr W. Simulation of human autosomal dominant retinitis pigmentosa in transgenic mice expressing a mutated murine opsin gene. Proceedings of the National Academy of Sciences. 1993;90(12):5499-503.

7. Sung CH, Davenport CM, Hennessey JC, Maumenee IH, Jacobson SG, Heckenlively JR, et al. Rhodopsin mutations in autosomal dominant retinitis pigmentosa. Proceedings of the National Academy of Sciences of the United States of America. 1991;88(15):6481-5.

8. Berson EL, Rosner B, Sandberg MA, Dryja TP. Ocular findings in patients with autosomal dominant retinitis pigmentosa and a rhodopsin gene defect (Pro-23-His). Arch Ophthalmol. 1991;109(1):92101.

9. Dryja TP, McGee TL, Reichel E, Hahn LB, Cowley GS, Yandell DW, et al. A point mutation of the rhodopsin gene in one form of retinitis pigmentosa. Nature. 1990;343(6256):364-6.

10. Chytil A, Magnuson MA, Wright CV, Moses HL. Conditional inactivation of the TGF-beta type II receptor using Cre:Lox. Genesis. 2002;32(2):73-5.

11. Kugler M, Schlecht A, Fuchshofer R, Schmitt SI, Kleiter I, Aigner L, et al. SMAD7 deficiency stimulates Müller progenitor cell proliferation during the development of the mammalian retina. Histochemistry and Cell Biology. 2017.

12. Richardson KC, Jarett L, Finke EH. Embedding in epoxy resins for ultrathin sectioning in electron microscopy. Stain Technology. 1960;35:313-23.

13. Braunger BM, Leimbeck SV, Schlecht A, Volz C, Jägle H, Tamm ER. Deletion of ocular transforming growth factor $\beta$ signaling mimics essential characteristics of diabetic retinopathy. The American Journal of Pathology. 2015;185(6):1749-68.

14. Boneva SK, Gross TR, Schlecht A, Schmitt SI, Sippl C, Jagle H, et al. Cre recombinase expression or topical tamoxifen treatment do not affect retinal structure and function, neuronal vulnerability or glial reactivity in the mouse eye. Neuroscience. 2016;325:188-201.

15. Braunger BM, OhImann A, Koch M, Tanimoto N, Volz C, Yang Y, et al. Constitutive overexpression of Norrin activates $\mathrm{Wnt} / \beta$-catenin and endothelin-2 signaling to protect photoreceptors from light damage. Neurobiology of disease. 2013;50:1-12.

16. Braunger BM, Pielmeier S, Demmer C, Landstorfer V, Kawall D, Abramov N, et al. TGF- $\beta$ Signaling Protects Retinal Neurons from Programmed Cell Death during the Development of the Mammalian Eye. Journal of Neuroscience. 2013;33(35):14246-58.

17. Schlecht A, Leimbeck SV, Jägle H, Feuchtinger A, Tamm ER, Braunger BM. Deletion of Endothelial Transforming Growth Factor- $\beta$ Signaling Leads to Choroidal Neovascularization. The American Journal of Pathology. 2017;187(11):2570-89.

18. Livak KJ, Schmittgen TD. Analysis of relative gene expression data using real-time quantitative PCR and the 2(-Delta Delta C(T)) Method. Methods. 2001;25(4):402-8.

19. Andrews S. FastQC A Quality Control tool for High Throughput Sequence Data. http://wwwbioinformaticsbabrahamacuk/projects/fastqc/. 
20. Dobin A, Davis CA, Schlesinger F, Drenkow J, Zaleski C, Jha S, et al. STAR: ultrafast universal RNAseq aligner. Bioinformatics. 2013;29(1):15-21.

21. Patro R, Duggal G, Love MI, Irizarry RA, Kingsford C. Salmon provides fast and bias-aware quantification of transcript expression. Nat Methods. 2017;14(4):417-9.

22. Soneson C, Love MI, Robinson MD. Differential analyses for RNA-seq: transcript-level estimates improve gene-level inferences. F1000Research. 2015;4:1521.

23. Love MI, Huber W, Anders S. Moderated estimation of fold change and dispersion for RNA-seq data with DESeq2. Genome biology. 2014;15(12):550.

24. Stephens M. False discovery rates: a new deal. Biostatistics. 2017;18(2):275-94.

25. Ritchie ME, Phipson B, Wu D, Hu Y, Law CW, Shi W, et al. limma powers differential expression analyses for RNA-sequencing and microarray studies. Nucleic Acids Res. 2015;43(7):e47.

26. Langfelder P, Horvath S. WGCNA: an R package for weighted correlation network analysis. BMC bioinformatics. 2008;9:559.

27. Langfelder $P$, Mischel PS, Horvath $S$. When is hub gene selection better than standard meta-analysis? PloS One. 2013;8(4):e61505.

28. Gu Z, Eils R, Schlesner M. Complex heatmaps reveal patterns and correlations in multidimensional genomic data. Bioinformatics. 2016;32(18):2847-9.

29. Wu G, Feng X, Stein L. A human functional protein interaction network and its application to cancer data analysis. Genome biology. 2010;11(5):R53.

30. Chen EY, Tan CM, Kou Y, Duan Q, Wang Z, Meirelles GV, et al. Enrichr: interactive and collaborative HTML5 gene list enrichment analysis tool. BMC Bioinformatics. 2013;14:128.

31. Kuleshov MV, Jones MR, Rouillard AD, Fernandez NF, Duan Q, Wang Z, et al. Enrichr: a comprehensive gene set enrichment analysis web server 2016 update. Nucleic Acids Res. 2016;44(W1):W90-7.

32. Joly S, Lange C, Thiersch M, Samardzija M, Grimm C. Leukemia inhibitory factor extends the lifespan of injured photoreceptors in vivo. The Journal of neuroscience. 2008;28(51):13765-74.

33. Rattner A, Nathans $\mathrm{J}$. The genomic response to retinal disease and injury: evidence for endothelin signaling from photoreceptors to glia. The Journal of Neuroscience. 2005;25(18):4540-9.

34. Sofroniew MV, Vinters HV. Astrocytes: biology and pathology. Acta Neuropathologica. 2010;119(1):735.

35. Bringmann A, Pannicke T, Grosche J, Francke M, Wiedemann P, Skatchkov SN, et al. Müller cells in the healthy and diseased retina. Progress in retinal and eye research. 2006;25(4):397-424.

36. Feng C, Wang X, Liu T, Zhang M, Xu G, Ni Y. Expression of CCL2 and its receptor in activation and migration of microglia and monocytes induced by photoreceptor apoptosis. Mol Vis. 2017;23:765-77.

37. Rutar M, Natoli R, Valter K, Provis JM. Early focal expression of the chemokine Ccl2 by Muller cells during exposure to damage-inducing bright continuous light. Invest Ophthalmol Vis Sci. 2011;52(5):2379-88. 
38. Lewis GP, Erickson PA, Kaska DD, Fisher SK. An immunocytochemical comparison of Müller cells and astrocytes in the cat retina. Experimental Eye Research. 1988;47(6):839-53.

39. Schmitt SI, Bielmeier CB, Braunger BM. New Insights into Endothelin Signaling and Its Diverse Roles in the Retina. Adv Exp Med Biol. 2019;1185:519-23.

40. Chen W-W, Zhang XIA, Huang W-J. Role of neuroinflammation in neurodegenerative diseases (Review). Mol Med Rep. 2016;13(4):3391-6.

41. Cuenca N, Fernandez-Sanchez L, Campello L, Maneu V, De la Villa P, Lax P, et al. Cellular responses following retinal injuries and therapeutic approaches for neurodegenerative diseases. Prog Retin Eye Res. 2014;43:17-75.

42. Stoll G, Jander S, Schroeter M. Cytokines in CNS disorders: neurotoxicity versus neuroprotection. J Neural Transm Suppl. 2000;59:81-9.

43. Hanisch U-K, Kettenmann H. Microglia: active sensor and versatile effector cells in the normal and pathologic brain. Nature Neuroscience. 2007;10(11):1387-94.

44. Langmann T. Microglia activation in retinal degeneration. Journal of Leukocyte Biology. 2007;81(6):1345-51.

45. Samardzija M, Wenzel A, Aufenberg S, Thiersch M, Reme C, Grimm C. Differential role of Jak-STAT signaling in retinal degenerations. FASEB J. 2006;20(13):2411-3.

46. Samardzija M, Wenzel A, Thiersch M, Frigg R, Reme C, Grimm C. Caspase-1 ablation protects photoreceptors in a model of autosomal dominant retinitis pigmentosa. Invest Ophthalmol Vis Sci. 2006;47(12):5181-90.

47. Rohrer B, Guo Y, Kunchithapautham K, Gilkeson GS. Eliminating Complement Factor D Reduces Photoreceptor Susceptibility to Light-Induced Damage. Investigative Ophthalmology \& Visual Science. 2007;48(11):5282-9.

48. Lohr HR, Kuntchithapautham K, Sharma AK, Rohrer B. Multiple, parallel cellular suicide mechanisms participate in photoreceptor cell death. Experimental Eye Research. 2006;83(2):380-9.

49. Rohrer B, Demos C, Frigg R, Grimm C. Classical complement activation and acquired immune response pathways are not essential for retinal degeneration in the rd1 mouse. Experimental eye research. 2007;84(1):82-91.

50. Rutar M, Natoli R, Provis JM. Small interfering RNA-mediated suppression of Ccl2 in Muller cells attenuates microglial recruitment and photoreceptor death following retinal degeneration. J Neuroinflammation. 2012;9:221.

51. Rutar M, Natoli R, Kozulin P, Valter K, Gatenby P, Provis JM. Analysis of Complement Expression in Light-Induced Retinal Degeneration: Synthesis and Deposition of C3 by Microglia/Macrophages Is Associated with Focal Photoreceptor Degeneration. Investigative Ophthalmology \& Visual Science. 2011;52(8):5347-58.

52. Hadziahmetovic M, Kumar U, Song Y, Grieco S, Song D, Li Y, et al. Microarray Analysis of Murine Retinal Light Damage Reveals Changes in Iron Regulatory, Complement, and Antioxidant Genes in 
the Neurosensory Retina and Isolated RPE. Investigative Ophthalmology \& Visual Science. 2012;53(9):5231-41.

53. Schäfer N, Grosche A, Schmitt SI, Braunger BM, Pauly D. Complement Components Showed a TimeDependent Local Expression Pattern in Constant and Acute White Light-Induced Photoreceptor Damage. Frontiers in Molecular Neuroscience. 2017;10.

54. Silverman SM, Ma W, Wang X, Zhao L, Wong WT. C3- and CR3-dependent microglial clearance protects photoreceptors in retinitis pigmentosa. J Exp Med. 2019;216(8):1925-43.

55. Saint-Geniez M, Maharaj ASR, Walshe TE, Tucker BA, Sekiyama E, Kurihara T, et al. Endogenous VEGF is required for visual function: evidence for a survival role on müller cells and photoreceptors. PloS One. 2008;3(11):e3554.

56. Penn JW, Grobbelaar AO, Rolfe KJ. The role of the TGF- $\beta$ family in wound healing, burns and scarring: a review. International Journal of Burns and Trauma. 2012;2(1):18-28.

57. Saika S. TGFbeta pathobiology in the eye. Laboratory investigation; a journal of technical methods and pathology. 2006;86(2):106-15.

58. Goumans M-J, Liu Z, ten Dijke P. TGF-beta signaling in vascular biology and dysfunction. Cell Research. 2009;19(1):116-27.

59. Goumans MJ, Mummery C. Functional analysis of the TGFbeta receptor/Smad pathway through gene ablation in mice. The International Journal of Developmental Biology. 2000;44(3):253-65.

60. Massagué J. G1 cell-cycle control and cancer. Nature. 2004;432(7015):298-306.

61. Tesseur I, Nguyen A, Chang B, Li L, Woodling NS, Wyss-Coray T, et al. Deficiency in neuronal TGF- $\beta$ signaling leads to nigrostriatal degeneration and activation of TGF- $\beta$ signaling protects against MPTP neurotoxicity in mice. The Journal of Neuroscience: The Official Journal of the Society for Neuroscience. 2017.

62. Giaume C, Kirchhoff F, Matute C, Reichenbach A, Verkhratsky A. Glia: the fulcrum of brain diseases. Cell death and differentiation. 2007;14(7):1324-35.

\section{Tables}

Table1: Enrichment analysis for dysregulated genes derived from the VPP RNAseq analysis. Enriched pathways and potential upstream regulators were predicted using the indicated databases. For gene ontology enrichment, only the top 5 non-redundant significantly enriched biological process terms are shown. The numbers following the terms are the combined score as calculated by Enrichr. Only terms with a combined score $>5$ were considered. 


\begin{tabular}{|c|c|c|c|}
\hline \multirow{2}{*}{$\begin{array}{l}\text { dysregulation } \\
\text { analysis }\end{array}$} & Enriched pathways & \multirow{2}{*}{$\begin{array}{l}\text { Gene ontology enrichment } \\
\text { (biological process 2018) }\end{array}$} & \multirow{2}{*}{$\begin{array}{l}\text { Potential } \\
\text { regulators } \\
\text { 1: ChEA } \\
2016,2: \\
\text { ENCODE } \\
\text { TF ChIP- } \\
\text { seq } 2015\end{array}$} \\
\hline & $\begin{array}{l}\text { 1: BioPlanet 2019, 2: } \\
\text { Reactome 2016, 3: NCI-Nature } \\
2016\end{array}$ & & \\
\hline \multirow[t]{4}{*}{$\begin{array}{l}4620 \\
\text { upregulated } \\
\text { genes } \\
\left(\mathrm{p}_{\mathrm{adj}}<0.05\right)\end{array}$} & \multirow{3}{*}{$\begin{array}{l}\text { 1: Platelet activation, signaling } \\
\text { and aggregation } 98.77, \text { Axon } \\
\text { guidance } 92.59, \text { TGF-beta } \\
\text { regulation of extracellular } \\
\text { matrix } 89.40 \text {, Integrin cell } \\
\text { surface interactions } 80.66, \\
\text { PI3K class IB pathway in } \\
\text { neutrophils } 69.39 \\
\text { 2: Platelet activation, signaling } \\
\text { and aggregation } 98.99 \text {, } \\
\text { Integrin cell surface } \\
\text { interactions } 84.30, \\
\text { Hemostasis } 73.18 \text {, EPH-Ephrin } \\
62.44 \text {, Axon guidance } 56.40\end{array}$} & \multirow[t]{4}{*}{$\begin{array}{l}\text { extracellular matrix organization } \\
93.21 \text {, neutrophil activation } \\
\text { involved in immune response } \\
78.28 \text {, cellular response to cytokine } \\
\text { stimulus } 72.40 \text {, regulation of cell } \\
\text { migration } 69.84, \text { vascular } \\
\text { endothelial growth factor receptor } \\
\text { signaling pathway } 68.22\end{array}$} & \multirow{3}{*}{$\begin{array}{l}\text { 1: SUZ12 } \\
\text { 268.86, } \\
\text { MTF2 } \\
\text { 160.06, } \\
\text { WT1 } \\
96.47 \\
\\
\text { 2: EZH2 } \\
98.33, \\
\text { EP300 } \\
96.32, \\
\text { MYOD1 } \\
30.74\end{array}$} \\
\hline & & & \\
\hline & & & \\
\hline & $\begin{array}{l}\text { 3: Integrin family cell surface } \\
\text { interactions } 61.52 \text {, S1P3 } \\
\text { pathway 54.91, CXCR4- } \\
\text { mediated signaling events } \\
\text { 53.30, LPA receptor mediated } \\
\text { events 52.65, S1P1 pathway } \\
48.70\end{array}$ & & \\
\hline \multirow[t]{4}{*}{$\begin{array}{l}4636 \\
\text { downregulated } \\
\text { genes } \\
\left(p_{a d j}<0.05\right)\end{array}$} & $\begin{array}{l}\text { 1: Messenger RNA processing } \\
\text { 141.05, Visual signal } \\
\text { transduction: rods } 57.92, \\
\text { Global genomic nucleotide } \\
\text { excision repair } 45.63 \text {, Mitotic } \\
\text { G2-G2/M phases } 42.06 \text {, RNA } \\
\text { polymerase II transcription }\end{array}$ & \multirow[t]{4}{*}{$\begin{array}{l}\text { mRNA processing } 168.47 \text {, DNA } \\
\text { repair } 93.72, \text { cilium assembly } \\
68.84, \text { termination of RNA } \\
\text { polymerase II transcription } 55.78, \\
\text { rhodopsin mediated signaling } \\
\text { pathway } 48.90\end{array}$} & $\begin{array}{l}\text { 1: CREM } \\
212.80 \\
\text { FOXO3 } \\
172.49 \\
\text { KDM5B } \\
152.04\end{array}$ \\
\hline & 40.22 & & \multirow{3}{*}{$\begin{array}{l}\text { 2: KAT2A } \\
233.47 \\
\text { GABPA } \\
199.23 \\
\text { E2F4 } \\
196.15\end{array}$} \\
\hline & $\begin{array}{l}\text { 2: Assembly of the primary } \\
\text { cilium } 107.65 \text {, mRNA Splicing - } \\
\text { Major Pathway } 73.55, \\
\text { Activation of the } \\
\text { phototransduction cascade } \\
\text { 69.44, DNA Repair } 61.73 \text {, Cell } \\
\text { Cycle } 58.51\end{array}$ & & \\
\hline & $\begin{array}{l}\text { 3: Visual signal transduction: } \\
\text { Rods } 57.92 \text {, Fanconi anemia } \\
\text { pathway 27.00, ATR signaling } \\
\text { pathway 16.33, Regulation of } \\
\text { Telomerase 13.58, ATM } \\
\text { pathway } 12.73 \text {, p38 MAPK } \\
\text { signaling pathway } 11.53\end{array}$ & & \\
\hline
\end{tabular}


Table2: Enrichment analysis for WGCNA modules derived from the VPP RNAseq analysis. Enriched pathways and potential upstream regulators were predicted using the indicated databases. For gene ontology enrichment, only the top 5 non-redundant significantly enriched biological process terms are shown. The numbers following the terms are the combined score as calculated by Enrichr. Only terms with a combined score $>5$ were considered. Terms in bold font were also identified in the enrichment analysis of the dysregulated gene lists (combined score $>5$ ). n.s. = no significant enrichment. 


\begin{tabular}{|c|c|c|c|}
\hline \multirow[t]{2}{*}{$\begin{array}{l}\text { WGCNA } \\
\text { module }\end{array}$} & Enriched pathways & \multirow[t]{2}{*}{$\begin{array}{l}\text { Gene ontology enrichment } \\
\text { (biological process 2018) }\end{array}$} & \multirow{2}{*}{$\begin{array}{l}\text { Potential } \\
\text { regulators } \\
\text { 1: ChEA } \\
2016,2: \\
\text { ENCODE } \\
\text { TF ChIP- } \\
\text { seq } 2015\end{array}$} \\
\hline & $\begin{array}{l}\text { 1: BioPlanet 2019, 2: Reactome 2016, } \\
\text { 3: NCl-Nature } 2016\end{array}$ & & \\
\hline \multirow[t]{3}{*}{$\begin{array}{l}\text { Pos1 } \\
\text { (7705 } \\
\text { genes) }\end{array}$} & $\begin{array}{l}\text { 1: Axon guidance } 36.18 \text {, T helper cell } \\
\text { surface molecules } 32.25 \text {, Platelet } \\
\text { activation, signaling and aggregation } \\
29.28 \text {, Alpha-V beta-3 integrin/ OPN } \\
\text { pathway } 27.52 \text {, PI3K class IB pathway } \\
\text { in neutrophils } 25.55\end{array}$ & \multirow{3}{*}{$\begin{array}{l}\text { extracellular matrix organization } \\
44.07 \text {, } \\
\text { sprouting angiogenesis } 27.06, \\
\text { ephrin receptor signaling pathway } \\
24.92 \text {, response to cytokine } 24.09, \\
\text { vascular endothelial growth factor } \\
\text { receptor signaling } 23.91\end{array}$} & $\begin{array}{l}\text { 1: SUZ12 } \\
67.13 \\
\text { MTF2 } \\
\text { 35.24, } \\
\text { JARID2 } \\
25.08\end{array}$ \\
\hline & $\begin{array}{l}\text { 2: Integrin cell surface interactions } \\
\text { 31.84, Platelet activation, signaling } \\
\text { and aggregation } 29.71 \text {, Ephrin } \\
\text { signaling } 27.88 \text {, Extracellular matrix } \\
\text { organization } 27.76 \text {, Signal } \\
\text { amplification } 24.50 \text {, Semaphorin } \\
\text { interactions } 20.52\end{array}$ & & \multirow[t]{2}{*}{$\begin{array}{l}\text { 2: EZH2 } \\
14.15 \\
\text { EP300 } \\
6.68\end{array}$} \\
\hline & $\begin{array}{l}\text { 3: Osteopontin-mediated events } \\
27.52 \text {, Beta3 integrin cell surface } \\
\text { interactions } 25.55 \text {, S1P2 pathway } \\
25.22 \text {, S1P3 pathway } 24.00 \text {, LPA } \\
\text { receptor mediated events } 20.74\end{array}$ & & \\
\hline \multirow[t]{3}{*}{$\begin{array}{l}\text { Pos2 } \\
\text { (560 } \\
\text { genes) }\end{array}$} & $\begin{array}{l}\text { 1: Respiratory electron transport } \\
\text { 148.50, Ketone body metabolism } \\
70.09 \text {, Cap-dependent translation } \\
\text { initiation } 50.22 \text {, Nef-mediated CD8 } \\
\text { downregulation } 42.88 \text {, Cytoplasmic } \\
\text { ribosomal proteins } 39.30\end{array}$ & \multirow{3}{*}{$\begin{array}{l}\text { respiratory electron transport chain } \\
114.12 \text {, SRP-dependent } \\
\text { cotranslational protein targeting to } \\
\text { membrane } 57.24 \text {, negative } \\
\text { regulation of peptide } 56.44 \text {, } \\
\text { negative regulation of membrane } \\
\text { potential } 53.81 \text {, negative regulation } \\
\text { of necroptotic process } 53.81\end{array}$} & \multirow{2}{*}{$\begin{array}{l}\text { 1: EKLF } \\
13.21 \\
\text { THRA } \\
7.02 \\
\text { GATA1 } \\
6.80 \\
\text { 2: HCFC1 } \\
6.41\end{array}$} \\
\hline & $\begin{array}{l}\text { 2: Respiratory electron transport } \\
\text { 128.38, Eukaryotic Translation } \\
\text { Elongation } 57.24, \text { Nef Mediated CD8 } \\
\text { Down-regulation } 42.88 \text {, Orexin and } \\
\text { neuropeptides FF and QRFP bind to } \\
\text { their respective receptors } 35.11 \text {, } \\
\text { Ketone body metabolism 35.11 }\end{array}$ & & \\
\hline & $\begin{array}{l}\text { 3: Validated nuclear estrogen receptor } \\
\text { alpha network } 15.80 \text {, JNK signaling in } \\
\text { the CD4+ TCR pathway } 14.61, \text { PDGF } \\
\text { receptor signaling network } 11.03, \\
\text { Alpha-synuclein signaling } 9.45 \text {, Visual } \\
\text { signal transduction: Cones } 6.22\end{array}$ & & \\
\hline $\begin{array}{l}\text { Pos3 } \\
\text { ( } 272 \\
\text { genes) }\end{array}$ & $\begin{array}{l}\text { 1: Adrenoceptors } 82.98, \\
\text { Phospholipase C delta-1 interactions } \\
\text { in phospholipid-associated cell } \\
\text { signalling } 39.93 \text {, Serotonin and } \\
\text { melatonin biosynthesis } 39.93, \\
\text { FGFR1b ligand binding and activation } \\
\text { 39.93, Pyrimidine biosynthesis } 31.12\end{array}$ & $\begin{array}{l}\text { spinal cord dorsal/ventral } \\
\text { patterning } 365.39 \text {, osteoblast } \\
\text { development } 97.81 \text {, positive } \\
\text { regulation of catenin import into } \\
\text { nucleus } 97.81 \text {, DNA replication- } \\
\text { dependent nucleosome }\end{array}$ & $\begin{array}{l}\text { 1: FOXP1 } \\
\text { 17.13, } \\
\text { BP1 6.21 } \\
\text { 2: n.s. }\end{array}$ \\
\hline
\end{tabular}


2: Adrenoceptors 82.98, Free fatty acid receptors 39.93, Arachidonate production from DAG 39.93, Serotonin and melatonin biosynthesis 39.93 , FGFR1b ligand binding and activation 31.12

3: Signaling events mediated by the Hedgehog family 22.30, IL4-mediated signaling events 11.14, IL23-mediated signaling events 9.57, Circadian rhythm pathway 8.25 , BMP receptor signaling 7.68
Neg1 1: Messenger RNA processing 59.45, Global genomic nucleotide excision (7248 repair 25.86, RNA polymerase II Cgenes) terminal domain phosphorylation and interaction with capping enzyme 22.43, Visual signal transduction: rods 21.50 , Non-coding RNA metabolism 19.92

2: Assembly of the primary cilium 57.92, Processing of Intronless PremRNAs 42.06, Processing of Capped Intron-Containing Pre-mRNA 37.93, Homologous DNA Pairing and Strand Exchange 33.07, Activation of the phototransduction cascade 30.84

3: Fanconi anemia pathway 21.85 , Visual signal transduction: Rods 21.50, ATR signaling pathway 5.94 , Regulation of Telomerase 5.36, p38 MAPK signaling pathway 5.30

Neg2 1: Signaling by FGFR1 fusion mutants 56.25, Activation of NOXA and (506 translocation to mitochondria 16.74, genes) Polo-like kinase 3 (PLK3) pathway 16.74, Cyclin B2-mediated events 16.74, Tachykinin receptors bind tachykinins 16.74

2: Signaling by cytosolic FGFR1 fusion mutants 61.27, Heme biosynthesis 25.15, Golgi Cisternae Pericentriolar Stack Reorganization 17.20, Defective ABCA3 causes pulmonary surfactant metabolism dysfunction type 3 (SMDP3) 16.74, Hyaluronan biosynthesis and export 16.74

3: PLK3 signaling events 16.74 , Canonical NF-kappaB pathway 7.46 , TNF receptor signaling pathway 5.68 , TRAIL signaling pathway 5.22 , organization 71.54 , septin ring assembly 39.82
mRNA processing 70.46, DNA repair 38.32, ciliary basal bodyplasma membrane docking 29.59, DNA-templated transcription, termination 26.90 , histone lysine demethylation 22.88
1: FOXO3 51.83, YY1 27.21, CREB1 23.28

2: KAT2A 105.06, GABPA 89.64, E2F4 56.05

left/right pattern formation 49.64, 1: n.s. viral RNA genome replication 21.96, DNA replication-independent 2: n.s. nucleosome organization 19.36, mRNA splice site selection 18.87, positive regulation of vascular smooth muscle cell proliferation 17.20 
Signaling mediated by p38-gamma and p38-delta 5.04

Neg3 1: Cytoplasmic ribosomal proteins 195.82, Spliceosomal assembly

(586 176.30, Translation 169.55, genes) Respiratory electron transport, ATP biosynthesis by chemiosmotic coupling, and heat production by uncoupling proteins 137.50, Apoptotic factor-mediated response 104.27

2: Eukaryotic Translation Initiation 251.77, Mitochondrial translation 208.25, Cytochrome c-mediated apoptotic response 170.73 , rRNA processing 135.64, Respiratory electron transport, ATP synthesis by chemiosmotic coupling, and heat production by uncoupling proteins 129.02

3: PLK3 signaling events 13.51, DNAPK pathway in nonhomologous end joining 10.86, HIV-1 Nef: Negative effector of Fas and TNF-alpha 7.31, C-MYC pathway 5.73

\section{Figures}

mitochondrial translation 175.43, 1 : mitochondrial electron transport, JARID1A ubiquinol to cytochrome c 125.37, 89.00, translation 123.40, rRNA metabolic ETS1 process 122.84 , activation of cysteine-type endopeptidase activity involved in apoptotic process by cytochrome c 104.27 EKLF 44.12

2: EP300 54.49 , GABPA 48.40, KAT2A 46.47 

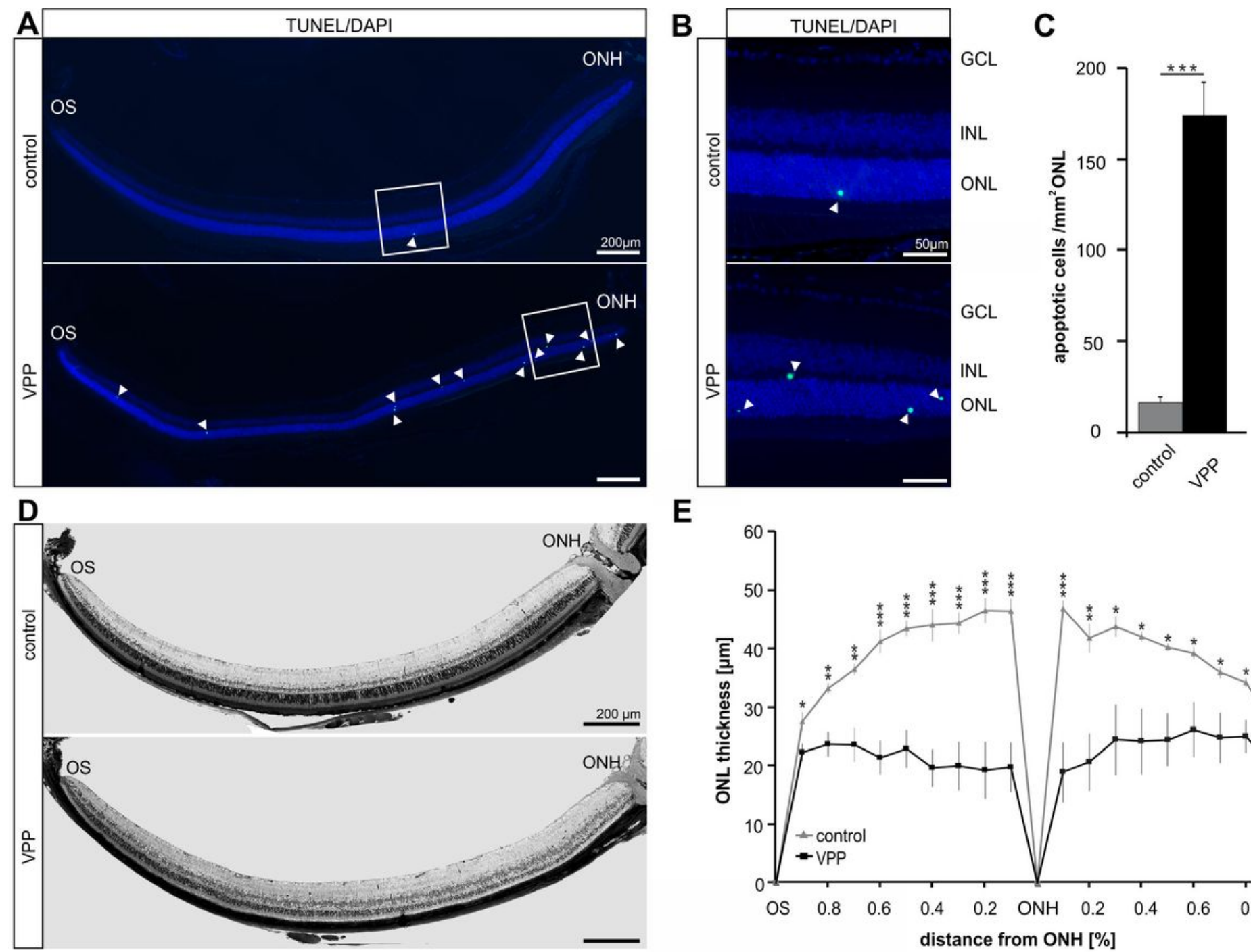

E
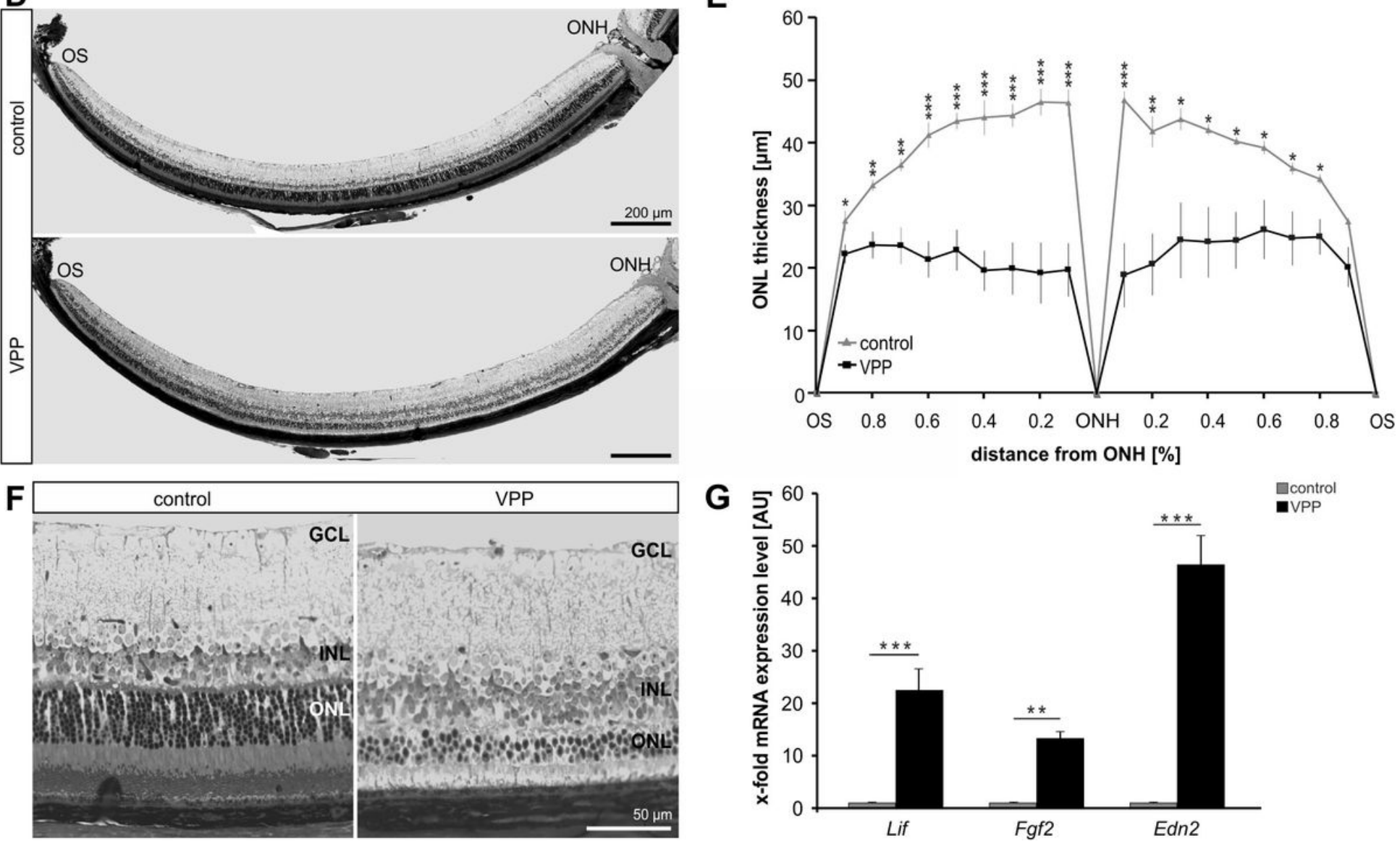

\section{Figure 1}

Apoptosis, retinal morphology/morphometry and the expression of neuroprotective factors in VPP and control mice A. and B. Mid-horizontal sections of one-month-old TdT-mediated dUTP-biotin nick end (TUNEL)-labeled retinae (A) and detailed magnification (B) of the boxed areas in (A). The VPP mouse showed more TUNEL-positive cells (green, arrowheads) in the ONL compared to its control littermate. Cell nuclei were stained with DAPI (blue). C. Total number TUNEL-positive cells normalized to mm2 ONL. Control $n=11$; VPP $n=11$. D. Richardson -stained, mid-horizontal semithin sections of three-month-old VPP and control mice. E and F. The detailed magnification of the central retina (F) shows the thinner ONL 
in the VPP animal compared to the ONL of the control. The thickness of the ONL was measured on midhorizontal semithin sections at defined measure points and the mean values were plotted in the spiderdiagram in (E) followed by statistical analysis. Controls $n=5$; VPP $n=5$. G. QPCR analyses for $m R N A$ of Lif, Fgf2 and Edn2 in three-month-old VPP and control retinae. The geometric mean value of the reference genes ubiquitin ( $\mathrm{Ubc}$ ) and guanine nucleotide binding protein subunit beta2 like 1 (Gnb2/1) was used for normalization. Controls: $\mathrm{n} \geq 4$; VPP: $\mathrm{n}=7$. $\mathrm{GCL}=$ ganglion cell layer; $\mathrm{INL}=$ inner nuclear layer; $\mathrm{ONL}=$ outer nuclear layer; $\mathrm{ONH}=$ optic nerve head; $\mathrm{OS}$ = ora serrata. Lif = leukaemia inhibitory factor, Fgf2 = fibroblast growth factor 2, Edn2 $=$ endothelin 2. Data are means \pm SEM. student`s t-test. ${ }^{*} p \leq 0.05, * \star p \leq$ $0.01, * \star \star p \leq 0.001$
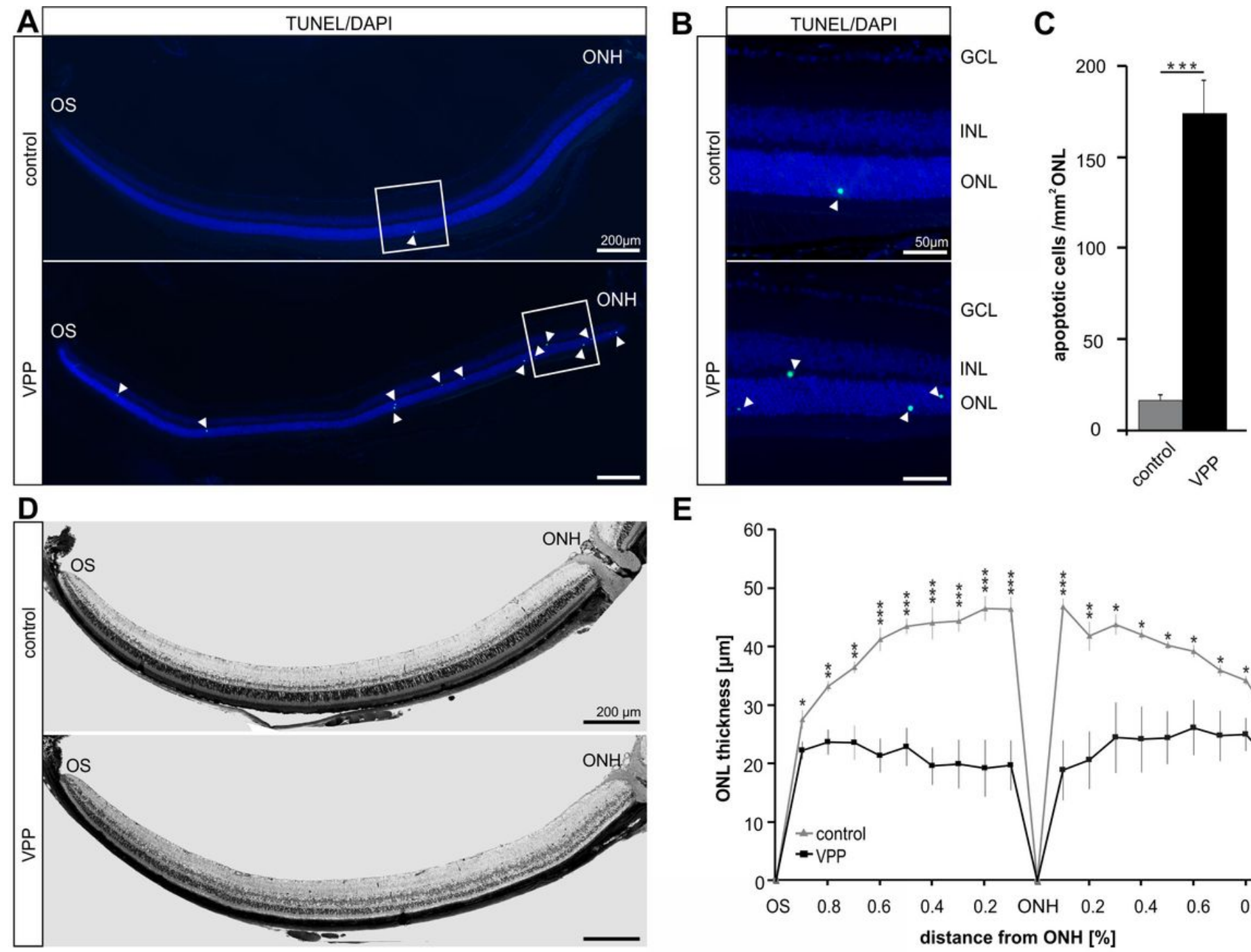

E
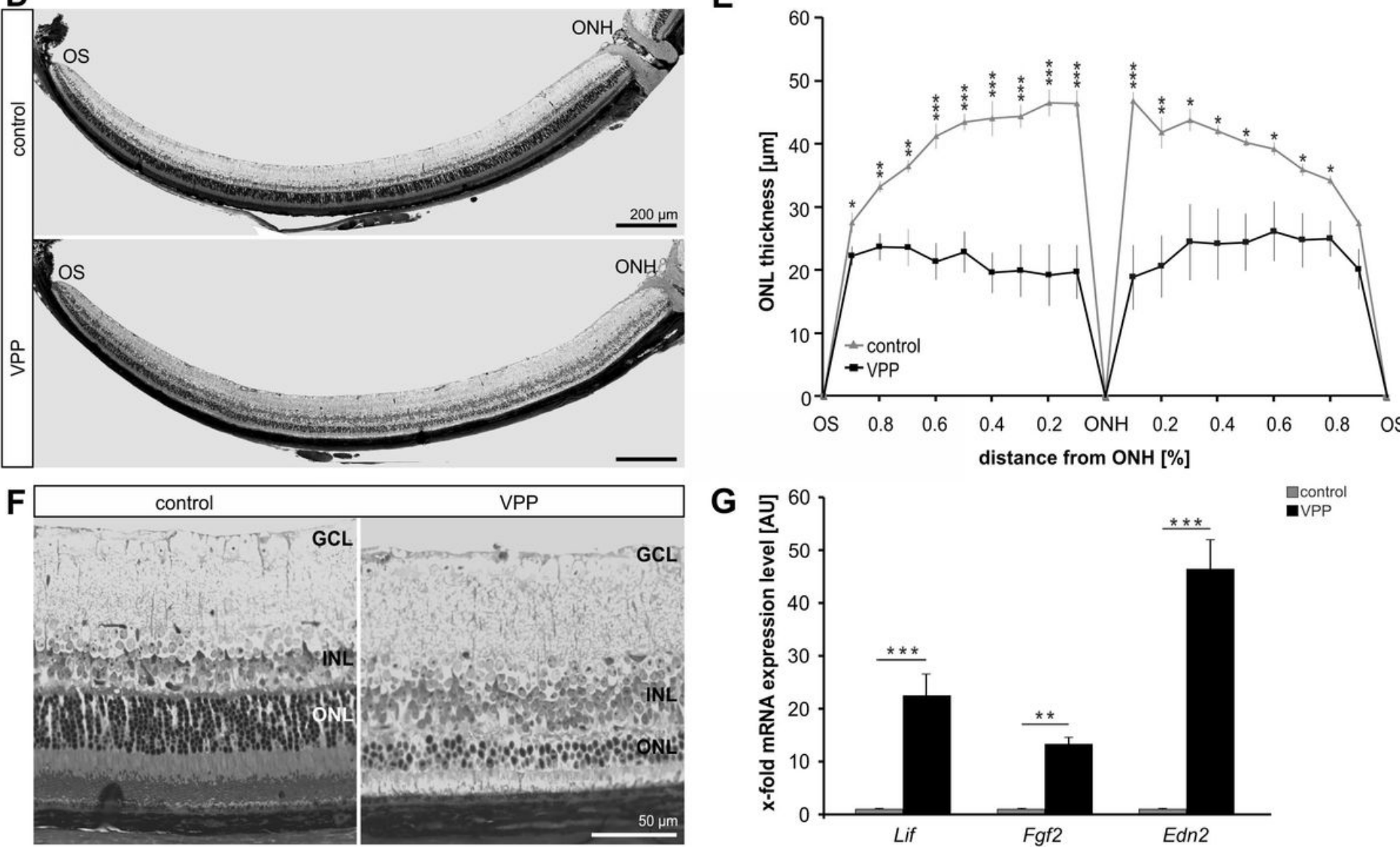


\section{Figure 1}

Apoptosis, retinal morphology/morphometry and the expression of neuroprotective factors in VPP and control mice A. and B. Mid-horizontal sections of one-month-old TdT-mediated dUTP-biotin nick end (TUNEL)-labeled retinae (A) and detailed magnification (B) of the boxed areas in (A). The VPP mouse showed more TUNEL-positive cells (green, arrowheads) in the ONL compared to its control littermate. Cell nuclei were stained with DAPI (blue). C. Total number TUNEL-positive cells normalized to $\mathrm{mm} 2 \mathrm{ONL}$. Control $n=11$; VPP $n=11$. D. Richardson -stained, mid-horizontal semithin sections of three-month-old VPP and control mice. E and F. The detailed magnification of the central retina (F) shows the thinner ONL in the VPP animal compared to the ONL of the control. The thickness of the ONL was measured on midhorizontal semithin sections at defined measure points and the mean values were plotted in the spiderdiagram in (E) followed by statistical analysis. Controls $n=5$; VPP $n=5$. G. QPCR analyses for $m R N A$ of Lif, Fgf2 and Edn2 in three-month-old VPP and control retinae. The geometric mean value of the reference genes ubiquitin ( $\mathrm{Ubc}$ ) and guanine nucleotide binding protein subunit beta2 like 1 (Gnb2/1) was used for normalization. Controls: $\mathrm{n} \geq 4$; VPP: $\mathrm{n}=7$. $\mathrm{GCL}=$ ganglion cell layer; $\mathrm{INL}=$ inner nuclear layer; $\mathrm{ONL}=$ outer nuclear layer; $\mathrm{ONH}=$ optic nerve head; $\mathrm{OS}$ = ora serrata. Lif = leukaemia inhibitory factor, Fgf2 = fibroblast growth factor $2, E d n 2=$ endothelin 2. Data are means \pm SEM. student's t-test. ${ }^{*} p \leq 0.05, * * p \leq$ $0.01, * * * \mathrm{p} \leq 0.001$.
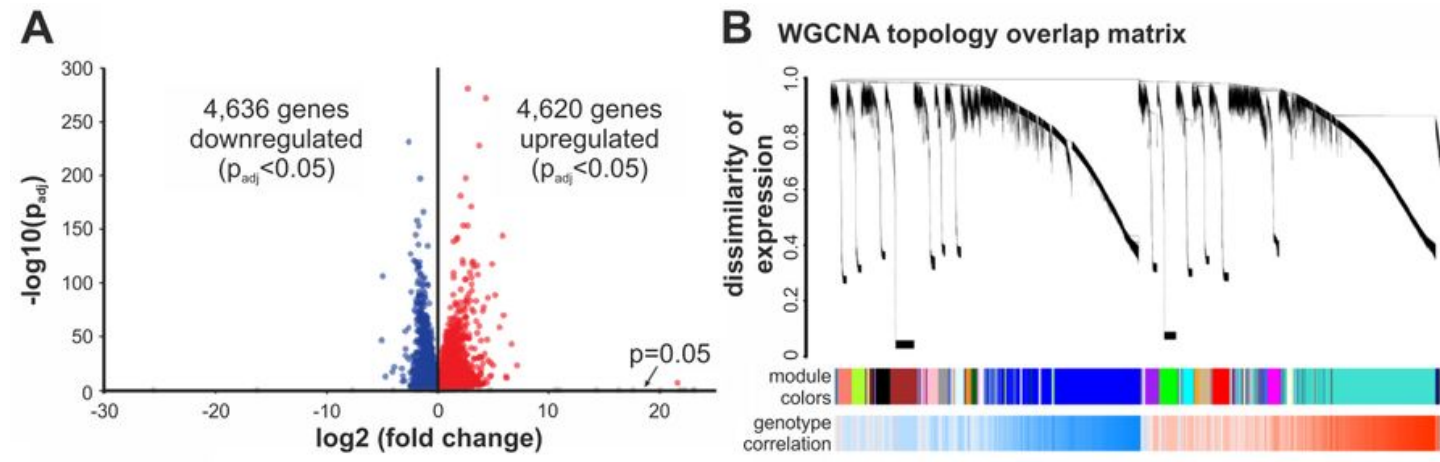

C WGCNA modules
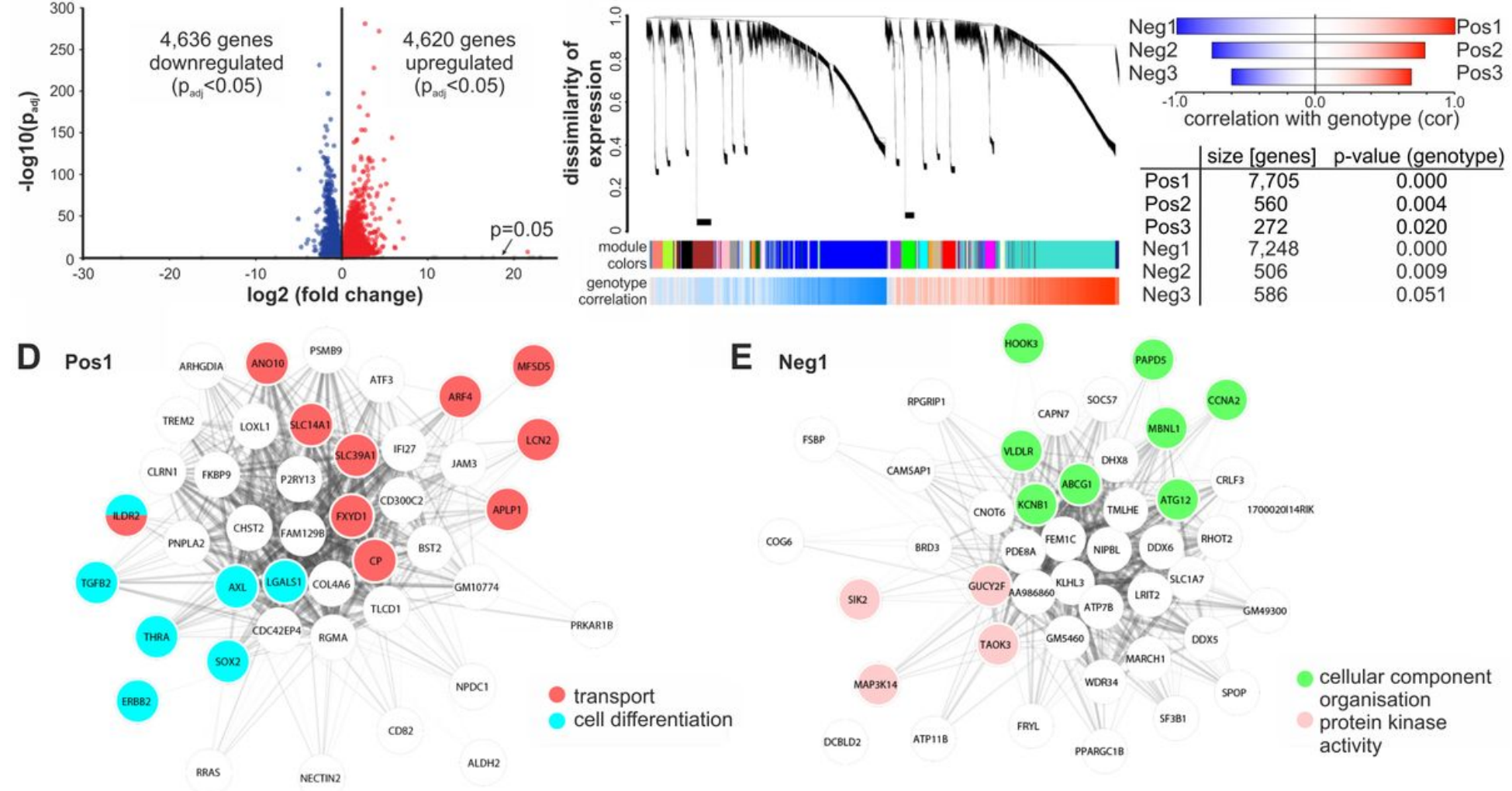

Figure 2

Transcriptome analysis of VPP mice A. RNAseq analysis of three-month-old retinae identified each more than 4600 significantly down- and up-regulated genes (Benjamini-Hochberg adjusted P-values; padj). B. 
WGCNA analysis showed large clusters of genes (modules) that were positively or negatively correlated with the genotype. Blue color in the panel below indicates lower expression and red color indicates higher expression in the VPP mice. C For each sign of correlation, three significantly correlated modules that changed in the VPP mice were identified. D. and E. Intra-module analysis of the Pos1 (D) and Neg1 (E) modules. The 50 highest connected (intramodular connectivity) genes are shown. Coloring of the genes corresponds to significantly enriched gene ontology terms.

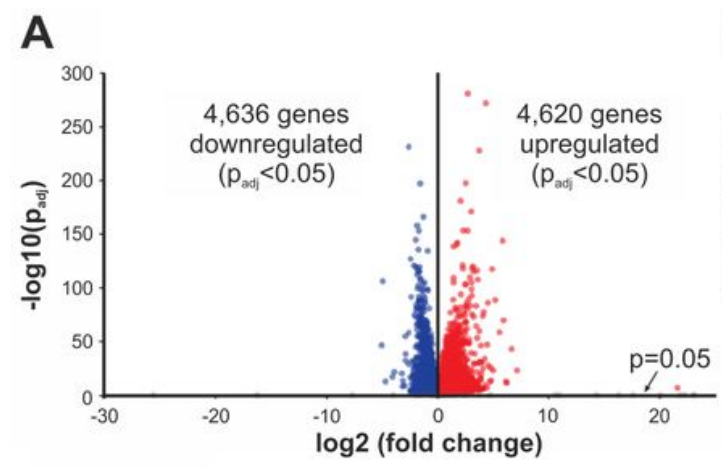

B WGCNA topology overlap matrix

C WGCNA modules
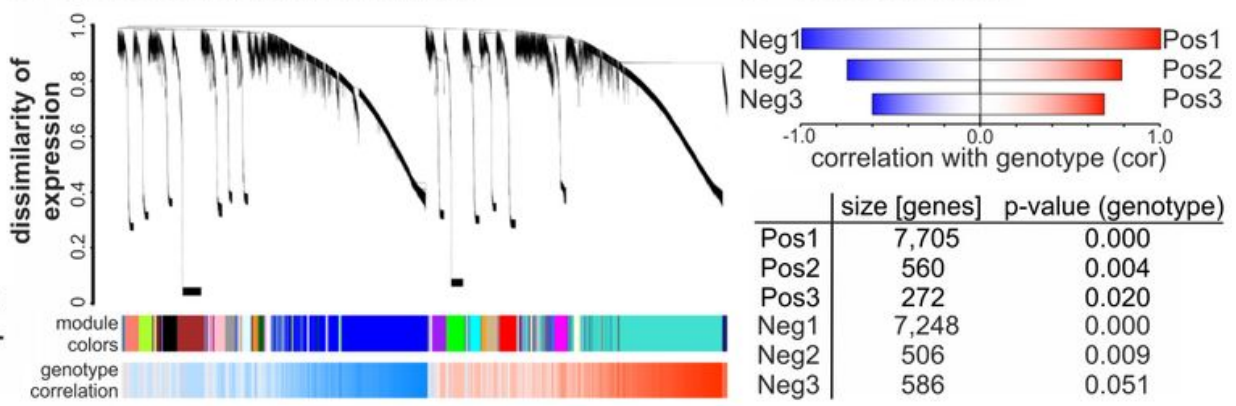

\section{Pos1}

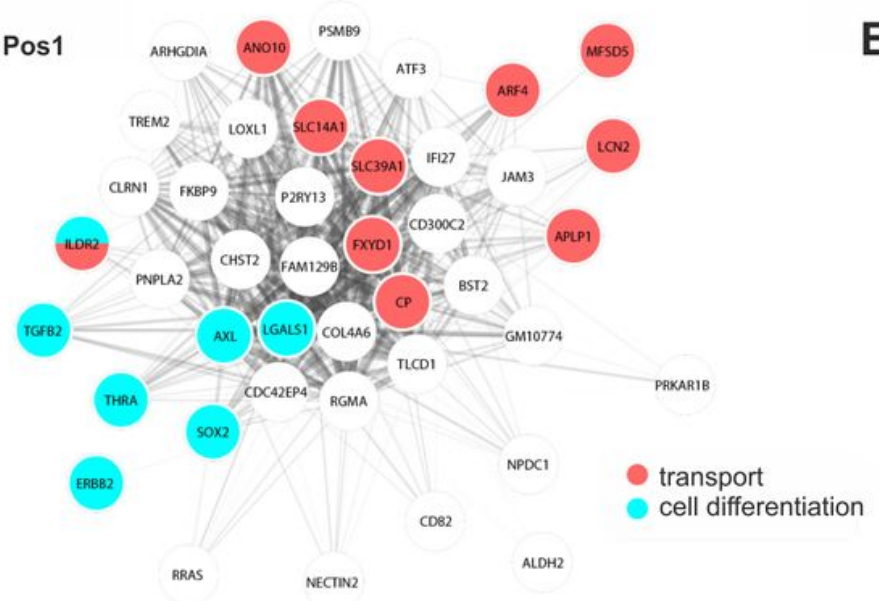

E Neg1

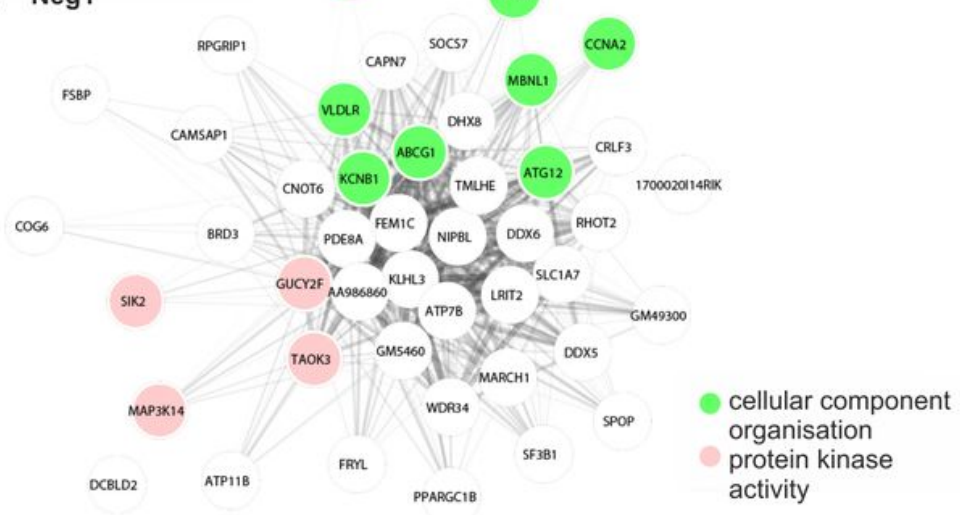

Figure 2

Transcriptome analysis of VPP mice A. RNAseq analysis of three-month-old retinae identified each more than 4600 significantly down- and up-regulated genes (Benjamini-Hochberg adjusted P-values; padj). B. WGCNA analysis showed large clusters of genes (modules) that were positively or negatively correlated with the genotype. Blue color in the panel below indicates lower expression and red color indicates higher expression in the VPP mice. C For each sign of correlation, three significantly correlated modules that changed in the VPP mice were identified. D. and E. Intra-module analysis of the Pos1 (D) and Neg1 (E) modules. The 50 highest connected (intramodular connectivity) genes are shown. Coloring of the genes corresponds to significantly enriched gene ontology terms. 

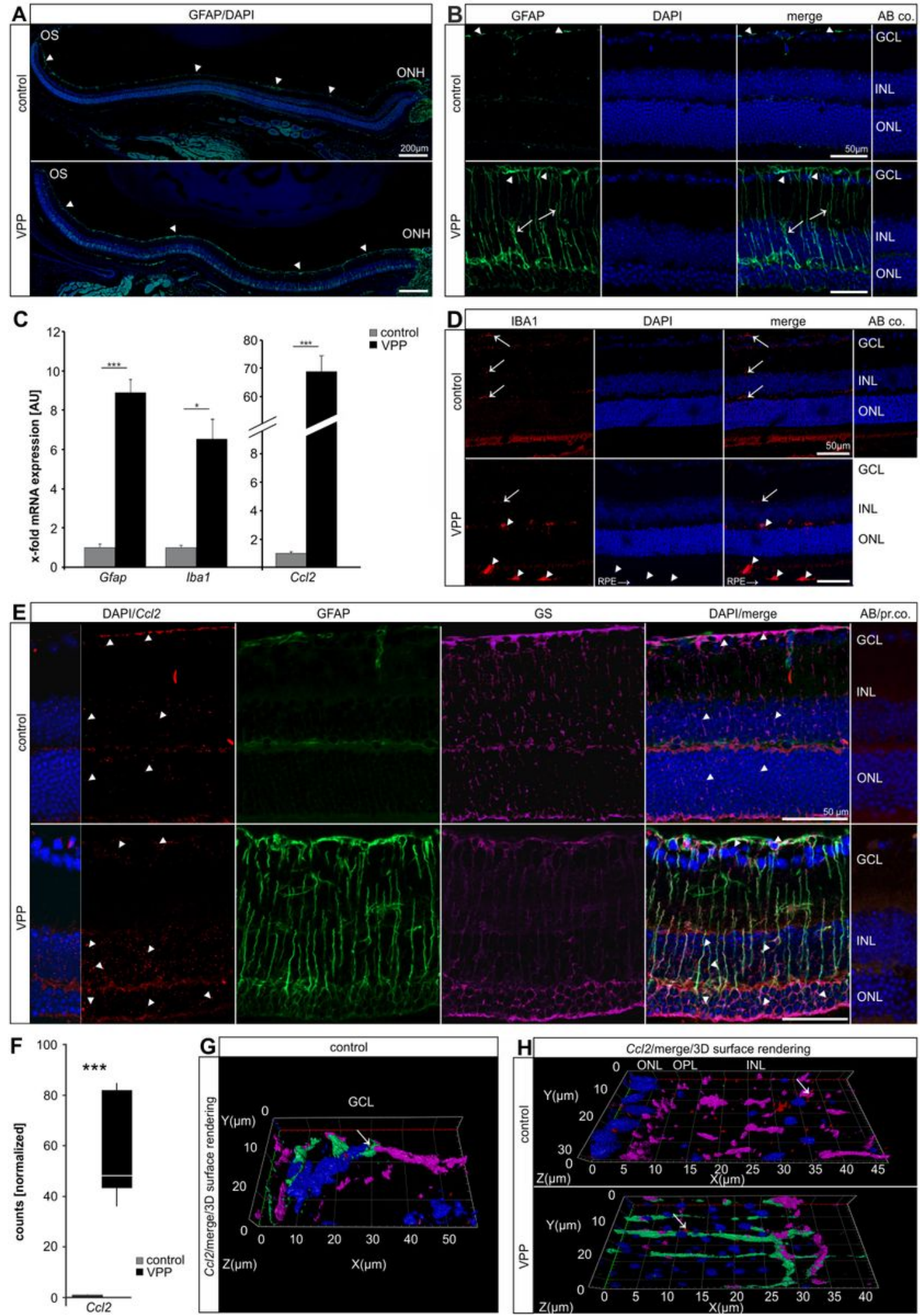

GS
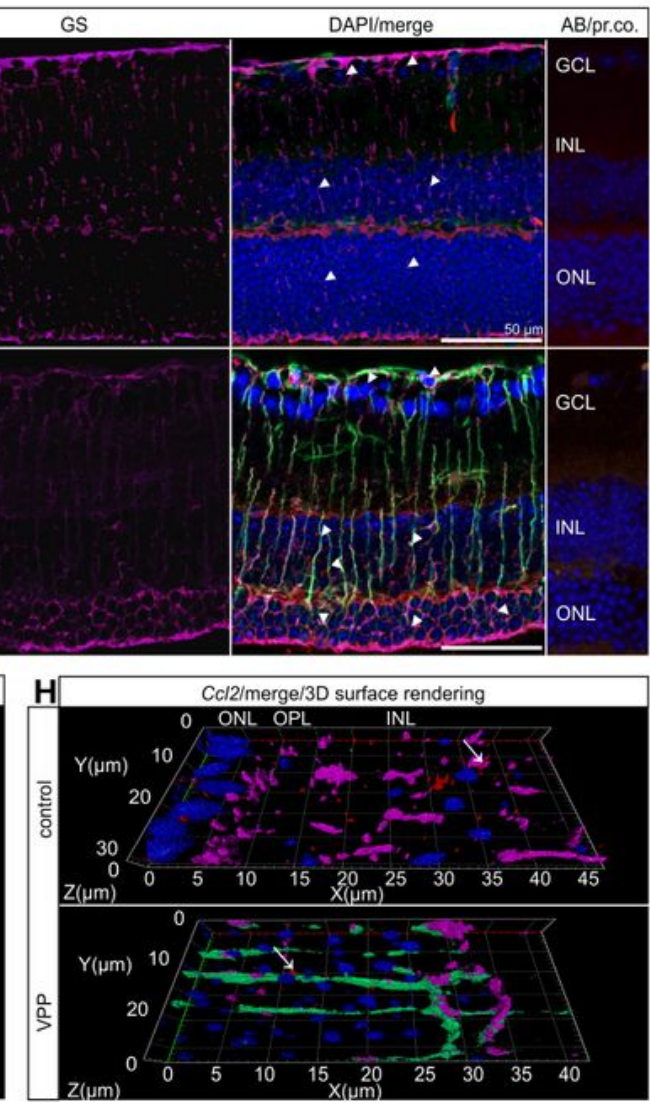

Figure 3

The glial response to photoreceptor degeneration in the VPP model A. Immunofluorescence staining against GFAP (green, arrowheads) on mid-horizontal sections of three-month-old eyes. GFAP was homogeneously distributed from the central to the peripheral retina. B. The detailed magnification showed the characteristic GFAP staining (green, arrowheads) pattern at the inner retinal surface in control and VPP animals. In the VPP retina, the labeling on the retinal surface was more intense and an 
additional radial staining pattern (arrows) was visible through the retina, indicating reactivity of Müller cells. Cell nuclei were DAPI-stained (blue). C. QPCR analyses for mRNA of retinal Gfap, Iba1 and Ccl2 in three-month-old animals. The geometric mean value of the reference genes ubiquitin (Ubc) and guanine nucleotide binding protein subunit beta2 like 1 (Gnb2/1) was used for normalization. Controls $n \geq 8$; VPP $\mathrm{n} \geq 7$. Data are means \pm SEM, student's t-test. ${ }^{*} \mathrm{p} \leq 0.05$, ${ }^{\star \star \star} \mathrm{p} \leq 0.001$. D. Immunoreactivity for IBA1 (red, arrows, arrowheads) in the retinae of one-month-old animals. Ramified IBA1-positive cells (arrows) were identified in the plexiform layers of the control retina. In the VPP retina, the IBA1-positive cells in the OPL were reactive, as indicated by their non-ramified phenotype (arrowheads) and non-ramified IBA1-positive cells (arrowheads) were visible in the sub-neuroretinal space in close proximity to the retinal pigment epithelium (RPE). Nuclei were DAPI-stained (blue). E. In situ hybridization for Ccl2 (red) and GFAP (green)/GS (purple) immunofluorescence co-labeling in the retinae of three-month-old animals. Nuclei were DAPI-stained (blue). In the control retina, a rather discrete Ccl2 signal (arrowheads) was visible in the ONL, INL and a few signals in the GCL. In the VPP retina, the number of the Ccl2 punctae increased (arrowheads) in the ONL and INL and the Müller cells were GFAP/GS-positive, indicating their reactivity. Ccl2 signals (red) were visible in the INL and ONL that did not overlap with GFAP/GS, indicating its further expression in neuronal cells. F. Boxplots showing the extracted $\mathrm{Ccl} 2$ expression data from the RNAseq as normalized counts for control and VPP genotypes. Control $n=6$; VPP $n=5$; padj $=8.05 \cdot 10-14$. G. and $\mathrm{H}$. Higher magnification of the GCL $(\mathrm{G})$ and $\mathrm{ONL} / \mathrm{OPL} / \mathrm{INL}$ region $(\mathrm{H})$ depictured as 3D reconstruction (Ccl2/merge/3D surface rendering). G. shows one of the few overlaps of the Ccl2 signals (red, arrow) with GFAP (green) - positive astrocytes. H. Ccl2 punctae (red, arrow) partially overlapped with GS (purple) positive resting (control animal, arrow) and GFAP (green)/ GS (purple) -positive reactive (VPP animal, arrow) Müller cells. GCL = ganglion cell layer; $\mathrm{INL}=$ inner nuclear layer; $\mathrm{OPL}=$ outer plexiform layer; $\mathrm{ONL}=$ outer nuclear layer; $\mathrm{RPE}=$ retinal pigment epithelium; $\mathrm{OS}$ = ora serrata; $\mathrm{ONH}=$ optic nerve head; $\mathrm{GFAP}=$ glial fibrillary acidic protein; IBA1 = ionized calcium-binding adapter molecule $1 ; \mathrm{Ccl} 2=\mathrm{CC}$-chemokine ligand 2; $G S$ = glutamine synthetase; $A B / p r$. co. = antibody $/$ probe control; $A U$ = arbitrary unit. 

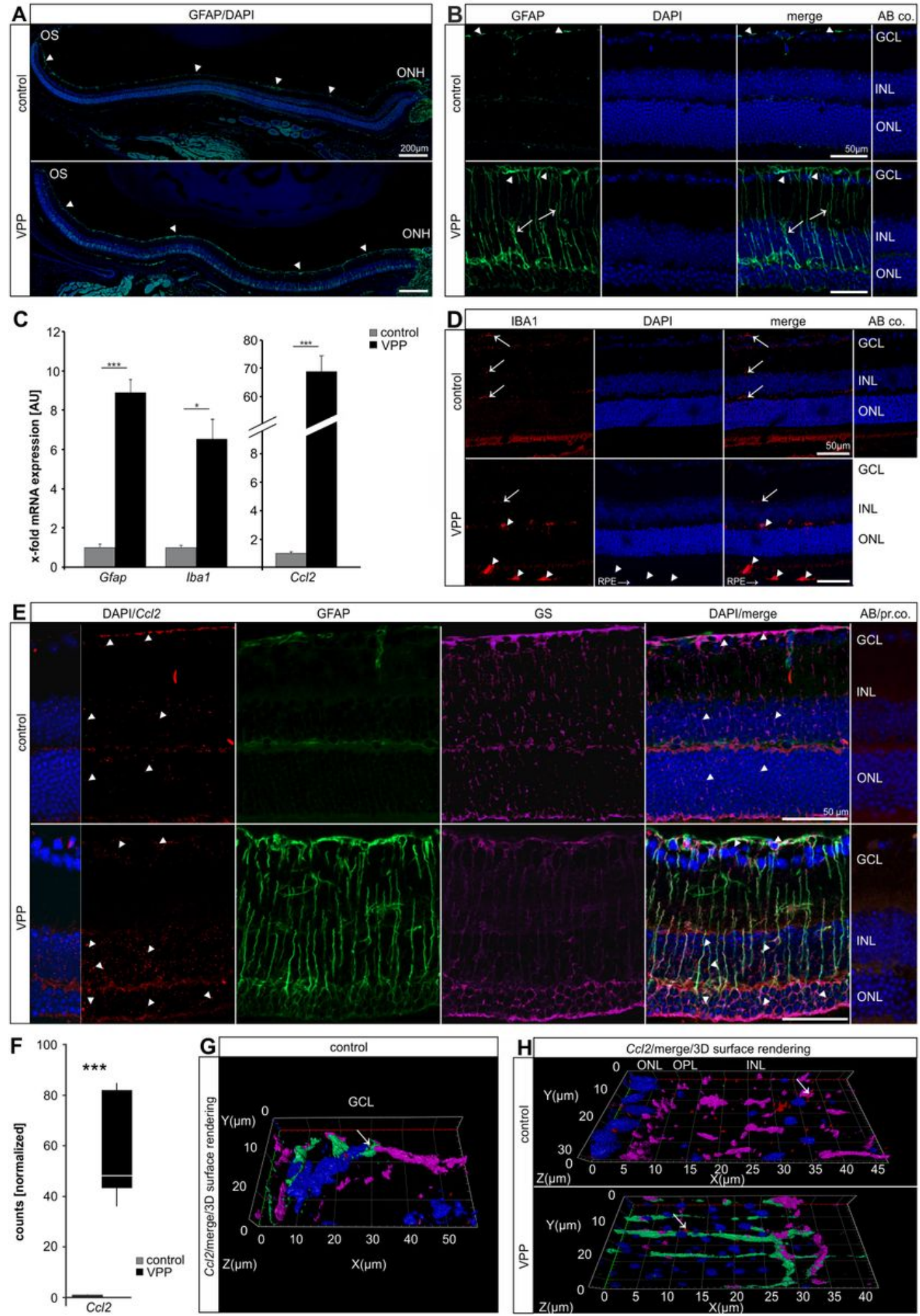

GS
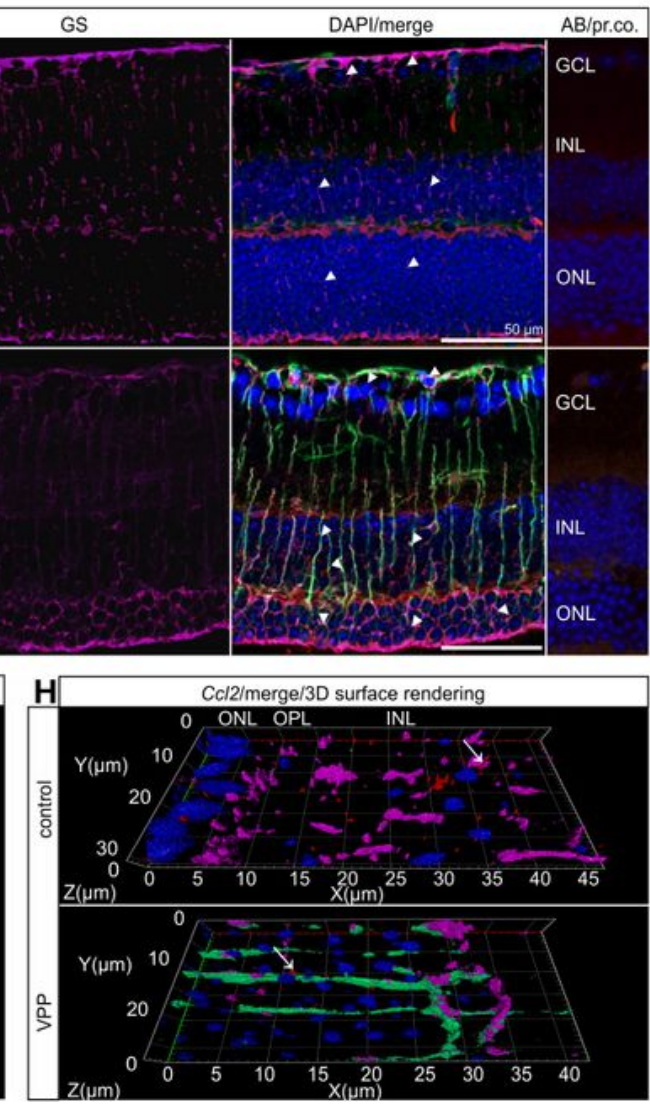

Figure 3

The glial response to photoreceptor degeneration in the VPP model A. Immunofluorescence staining against GFAP (green, arrowheads) on mid-horizontal sections of three-month-old eyes. GFAP was homogeneously distributed from the central to the peripheral retina. B. The detailed magnification showed the characteristic GFAP staining (green, arrowheads) pattern at the inner retinal surface in control and VPP animals. In the VPP retina, the labeling on the retinal surface was more intense and an 
additional radial staining pattern (arrows) was visible through the retina, indicating reactivity of Müller cells. Cell nuclei were DAPI-stained (blue). C. QPCR analyses for mRNA of retinal Gfap, Iba1 and Ccl2 in three-month-old animals. The geometric mean value of the reference genes ubiquitin (Ubc) and guanine nucleotide binding protein subunit beta2 like 1 (Gnb2/1) was used for normalization. Controls $n \geq 8$; VPP $\mathrm{n} \geq 7$. Data are means \pm SEM, student's t-test. ${ }^{*} \mathrm{p} \leq 0.05$, ${ }^{\star \star \star} \mathrm{p} \leq 0.001$. D. Immunoreactivity for IBA1 (red, arrows, arrowheads) in the retinae of one-month-old animals. Ramified IBA1-positive cells (arrows) were identified in the plexiform layers of the control retina. In the VPP retina, the IBA1-positive cells in the OPL were reactive, as indicated by their non-ramified phenotype (arrowheads) and non-ramified IBA1-positive cells (arrowheads) were visible in the sub-neuroretinal space in close proximity to the retinal pigment epithelium (RPE). Nuclei were DAPI-stained (blue). E. In situ hybridization for Ccl2 (red) and GFAP (green)/GS (purple) immunofluorescence co-labeling in the retinae of three-month-old animals. Nuclei were DAPI-stained (blue). In the control retina, a rather discrete Ccl2 signal (arrowheads) was visible in the ONL, INL and a few signals in the GCL. In the VPP retina, the number of the Ccl2 punctae increased (arrowheads) in the ONL and INL and the Müller cells were GFAP/GS-positive, indicating their reactivity. Ccl2 signals (red) were visible in the INL and ONL that did not overlap with GFAP/GS, indicating its further expression in neuronal cells. F. Boxplots showing the extracted $\mathrm{Ccl} 2$ expression data from the RNAseq as normalized counts for control and VPP genotypes. Control $n=6$; VPP $n=5$; padj $=8.05 \cdot 10-14$. G. and $\mathrm{H}$. Higher magnification of the GCL $(\mathrm{G})$ and $\mathrm{ONL} / \mathrm{OPL} / \mathrm{INL}$ region $(\mathrm{H})$ depictured as 3D reconstruction (Ccl2/merge/3D surface rendering). G. shows one of the few overlaps of the Ccl2 signals (red, arrow) with GFAP (green) - positive astrocytes. H. Ccl2 punctae (red, arrow) partially overlapped with GS (purple) positive resting (control animal, arrow) and GFAP (green)/ GS (purple) -positive reactive (VPP animal, arrow) Müller cells. GCL = ganglion cell layer; $\mathrm{INL}=$ inner nuclear layer; $\mathrm{OPL}=$ outer plexiform layer; $\mathrm{ONL}=$ outer nuclear layer; $\mathrm{RPE}=$ retinal pigment epithelium; $\mathrm{OS}$ = ora serrata; $\mathrm{ONH}=$ optic nerve head; $\mathrm{GFAP}=$ glial fibrillary acidic protein; IBA1 = ionized calcium-binding adapter molecule $1 ; \mathrm{Ccl} 2=\mathrm{CC}$-chemokine ligand 2; $G S$ = glutamine synthetase; $A B / p r$. co. = antibody $/$ probe control; $A U$ = arbitrary unit. 
A

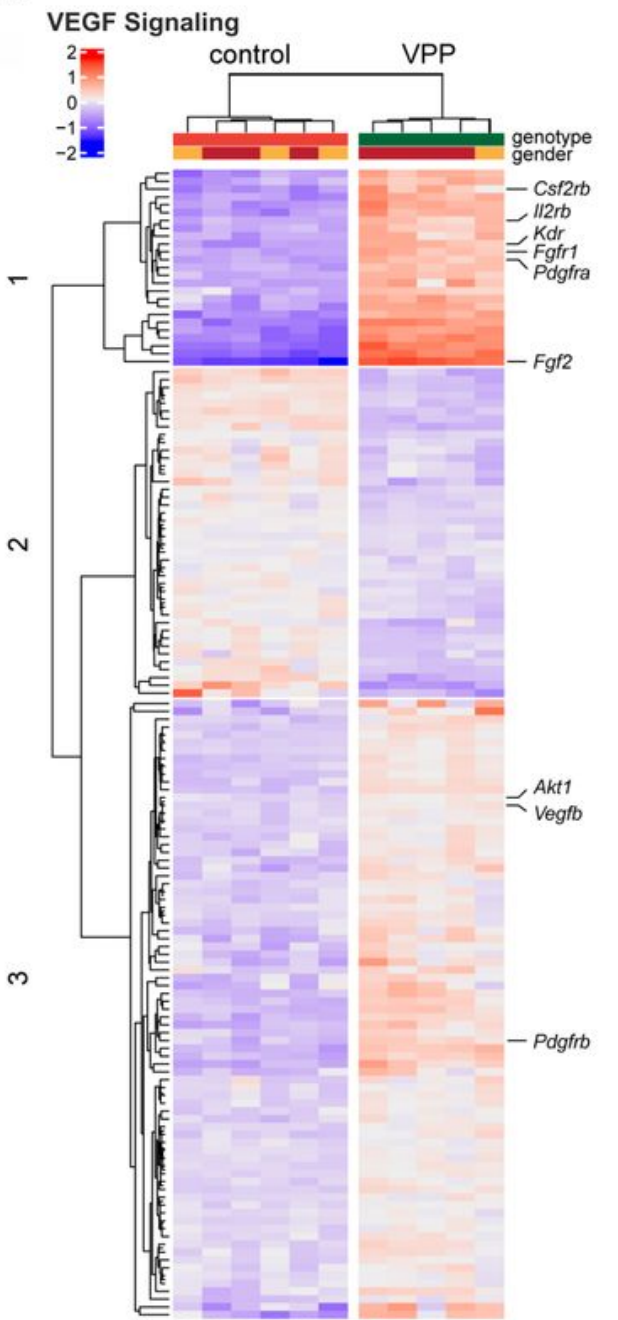

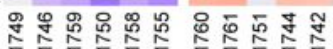

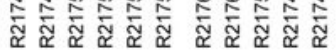

E

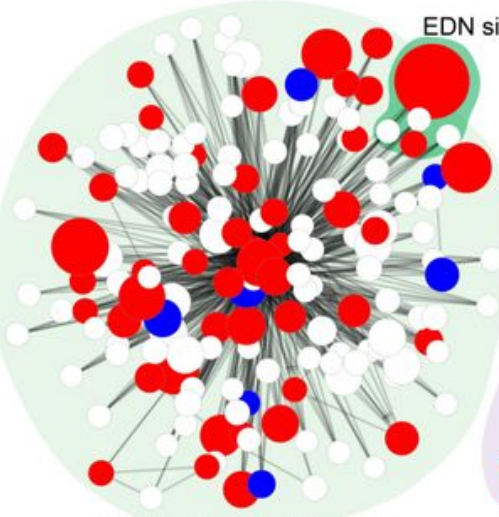

GPRC - $G$ alpha (q) signaling
B

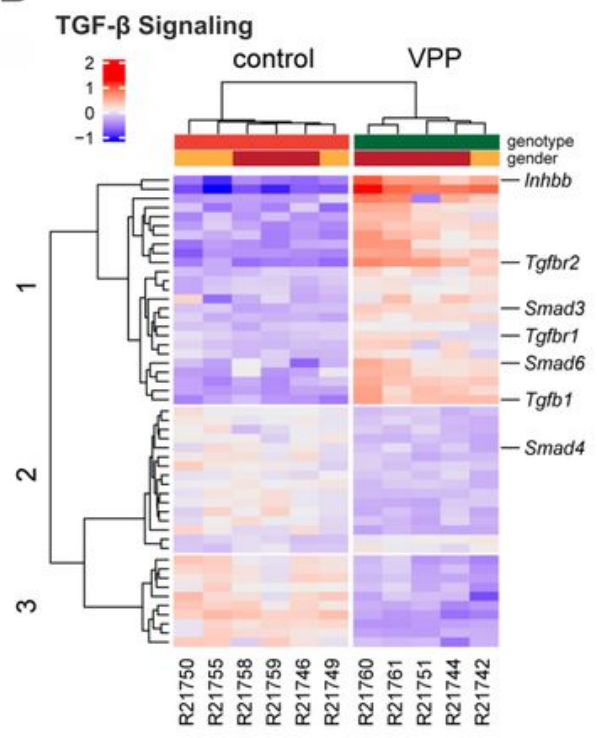

D

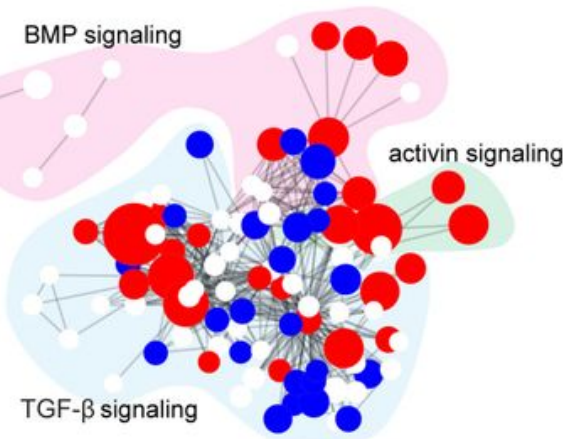

A

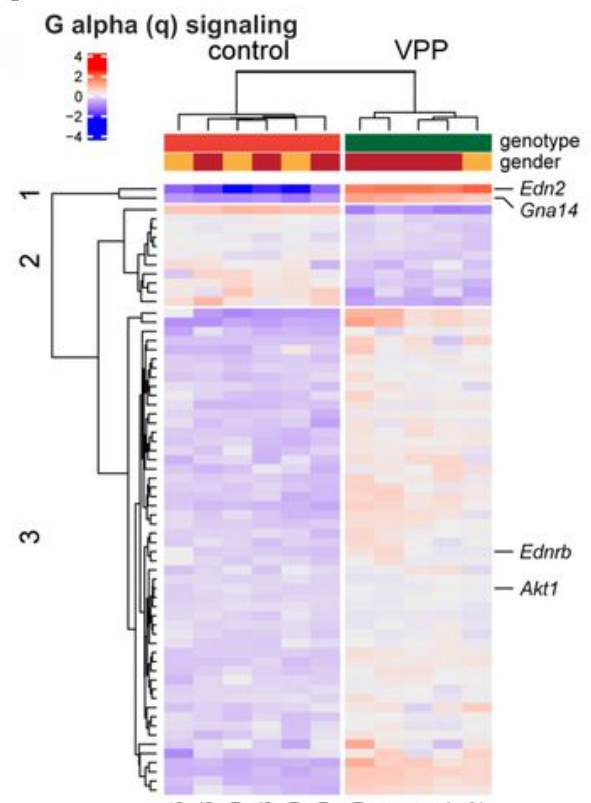

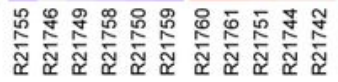
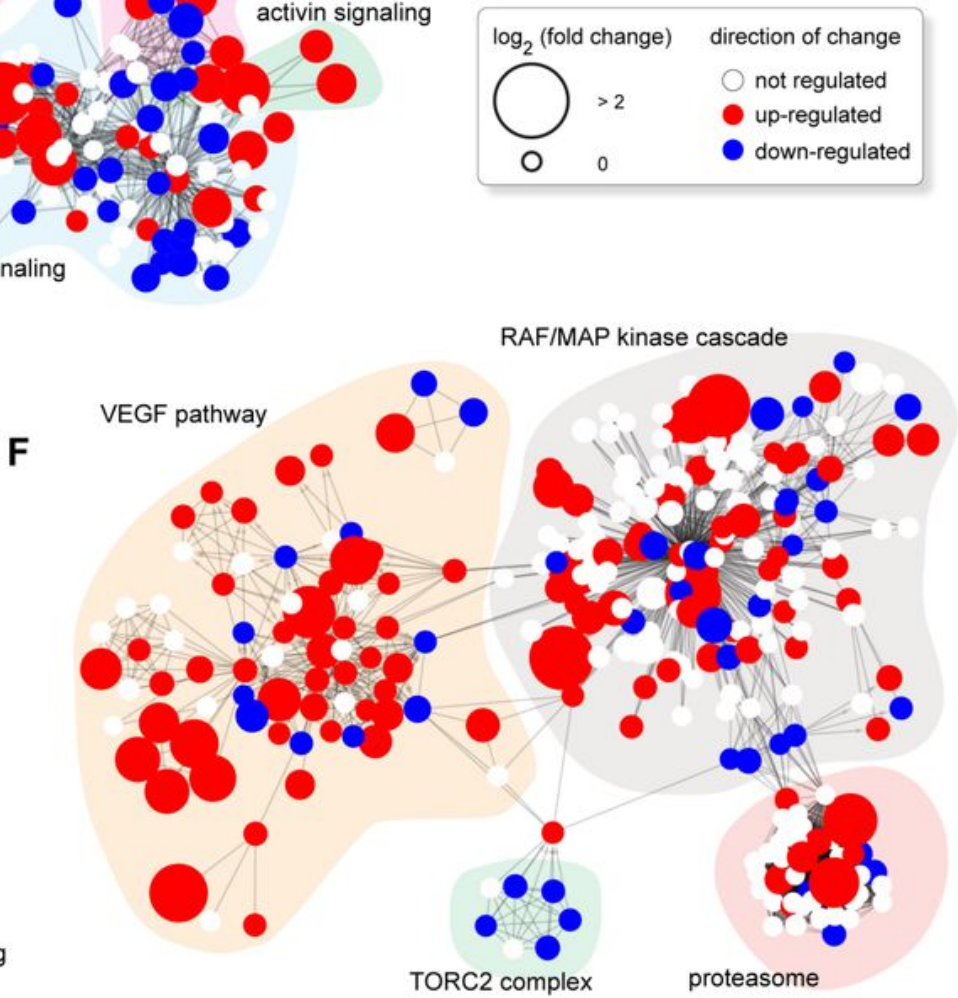

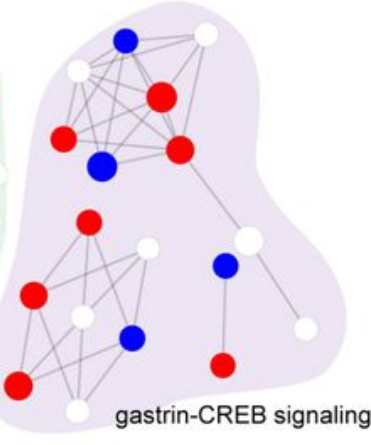

gastrin-CREB signaling

TORC2 complex

proteasome

\section{Figure 4}

Pathway analyses of transcriptomic changes in VPP mice A - C Heatmaps showing the significantly dysregulated genes in the Reactome pathways VEGF signaling (A), TGF- $\beta$ signaling (B) and G alpha (q) signaling (C). For each heatmap, the genotypes separate perfectly, as indicated by the unsupervised clustering above the heatmaps. Colors (red: upregulated, blue: downregulated) represent the deviation of the mean expression for each gene, independent of genotype. K-mer analysis into 3 groups revealed 
clusters of tightly co-regulated genes. Some interesting genes (e.g. neuroprotective or immune modulating function, directly involved in the intracellular signaling) are highlighted on the right. To further visualize sub-groups of pathways that were dysregulated, we converted the Reactome pathways into functional interaction networks. For each network genes were colored according to their dysregulation state: white - not significantly dysregulated; red - significantly upregulated and blue - significantly downregulated. The size of the nodes corresponds to the log2-fold change of regulation. The network for TGF-beta signaling is shown in D, G alpha (q) signaling is shown in E and VEGF signaling is shown in $F$. R21742-61 = RNAseq sample number 
A

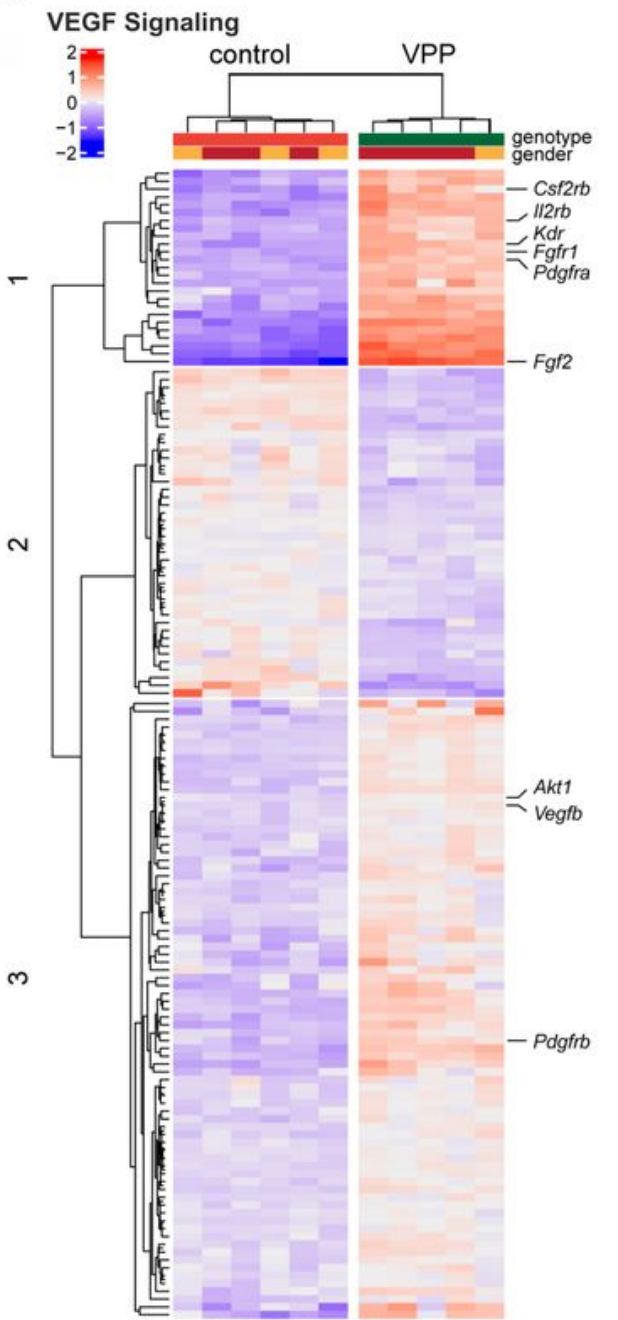

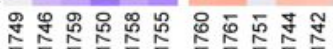

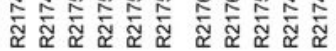

E

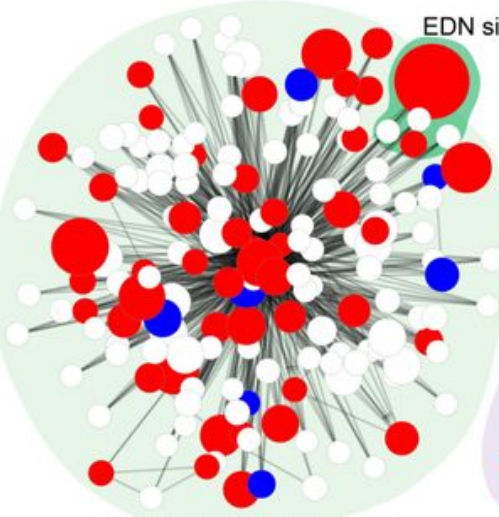

GPRC - $G$ alpha (q) signaling
B

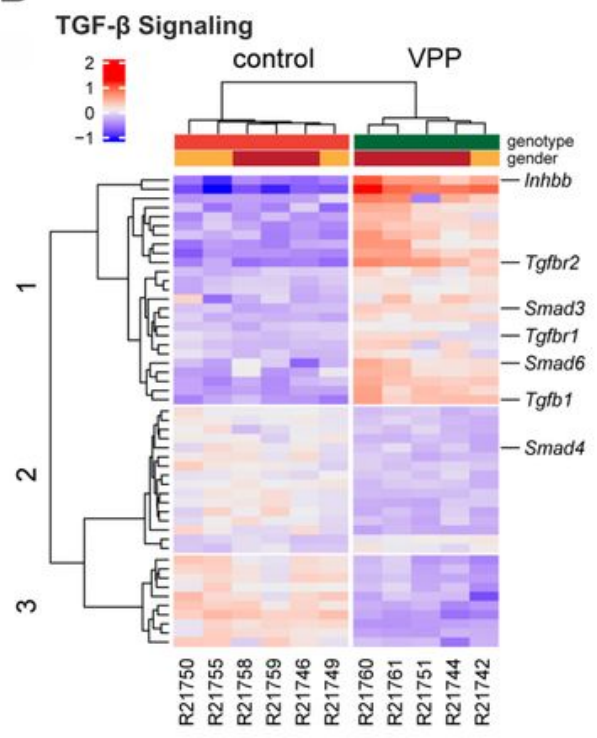

D

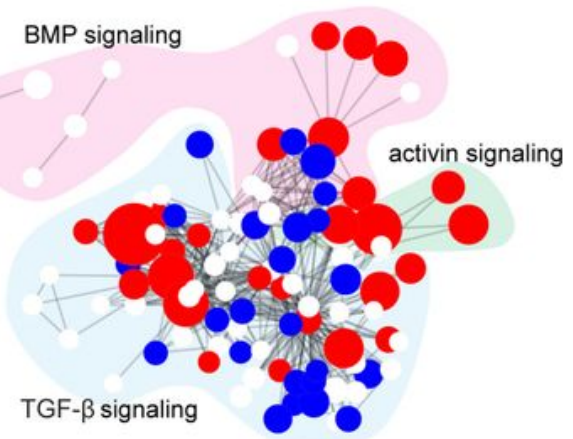

A

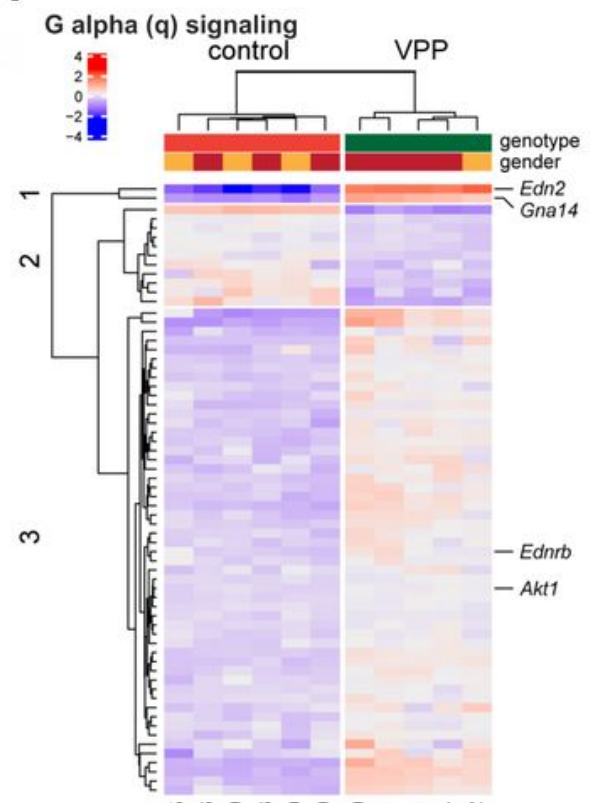

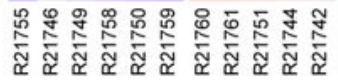
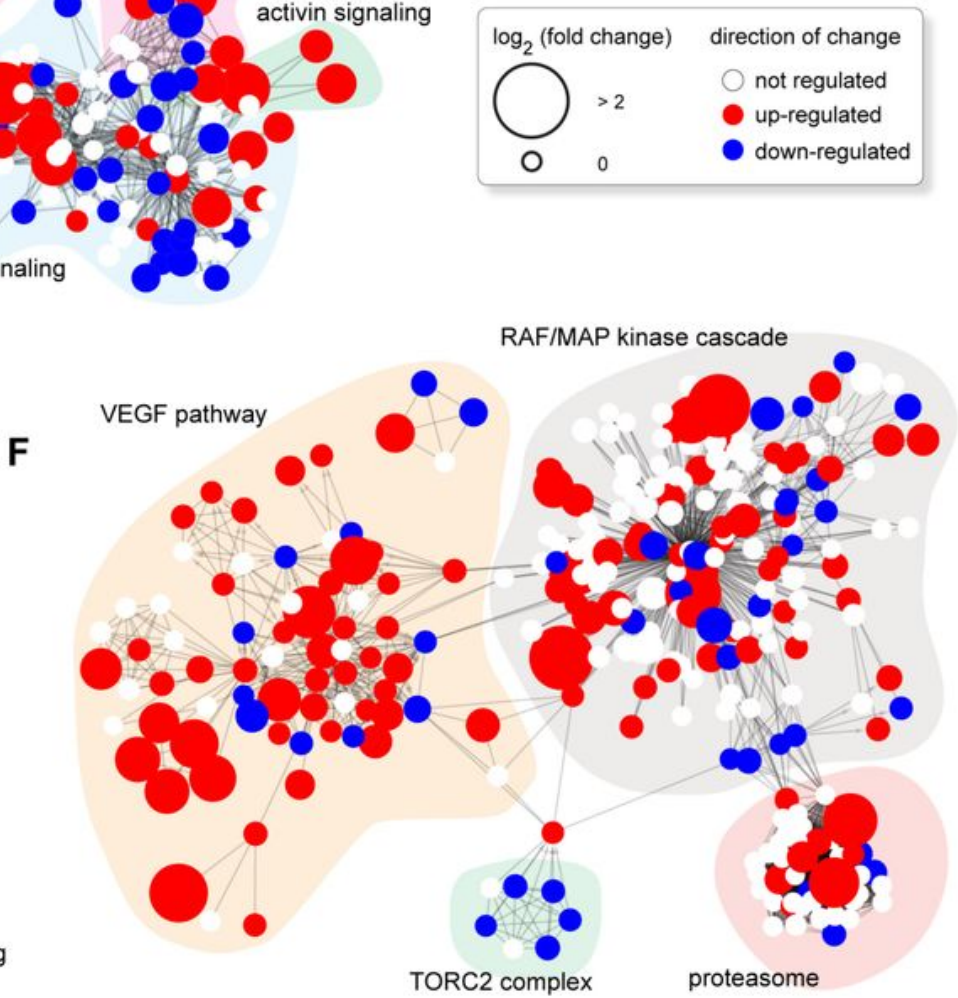

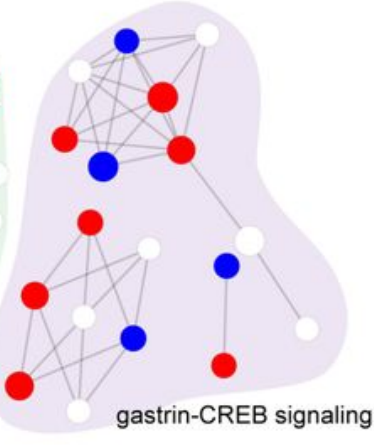

gastrin-CREB signaling

TORC2 complex

proteasome

\section{Figure 4}

Pathway analyses of transcriptomic changes in VPP mice A - C Heatmaps showing the significantly dysregulated genes in the Reactome pathways VEGF signaling (A), TGF- $\beta$ signaling (B) and G alpha (q) signaling (C). For each heatmap, the genotypes separate perfectly, as indicated by the unsupervised clustering above the heatmaps. Colors (red: upregulated, blue: downregulated) represent the deviation of the mean expression for each gene, independent of genotype. K-mer analysis into 3 groups revealed 
clusters of tightly co-regulated genes. Some interesting genes (e.g. neuroprotective or immune modulating function, directly involved in the intracellular signaling) are highlighted on the right. To further visualize sub-groups of pathways that were dysregulated, we converted the Reactome pathways into functional interaction networks. For each network genes were colored according to their dysregulation state: white - not significantly dysregulated; red - significantly upregulated and blue - significantly downregulated. The size of the nodes corresponds to the log2-fold change of regulation. The network for TGF-beta signaling is shown in D, G alpha (q) signaling is shown in E and VEGF signaling is shown in $F$. R21742-61 = RNAseq sample number 

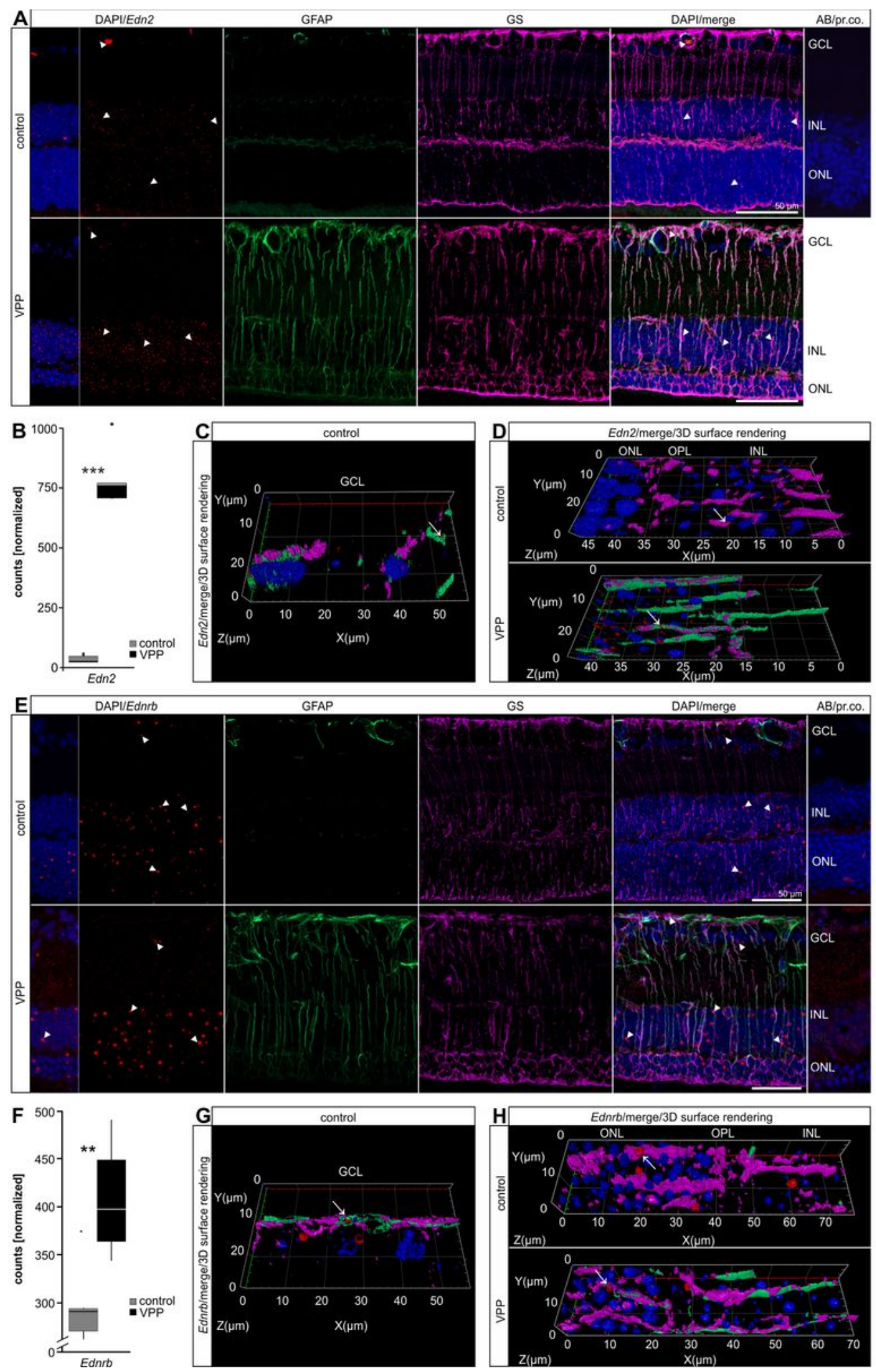

Figure 5

Upregulation of endothelin signaling in VPP mice A. In situ hybridization for Edn2 (red) and GFAP (green) /GS (purple) immunofluorescence co-labeling in the retinae of three-month-old animals. Nuclei were DAPIstained (blue). Edn2 (red, arrowheads) signals were detectable in the ONL and INL with some sparse signals in the GCL. In the VPP retina, the number of the Edn2 signals (red, arrowheads) was increased in the ONL and INL and the Müller cells were GFAP (green) /GS (purple) -positive, indicating their reactivity. 
Edn2 signals (red) were visible in the INL and ONL that did not overlap with GFAP/ GS, indicating its additional expression in neuronal cells. B. Boxplots showing the extracted Edn2 expression data from the RNAseq as normalized counts for control and VPP genotypes. Control $n=6$; VPP $n=5 ;$ padj $=8.68 \cdot 10-87$. C. and D. Higher magnification of the GCL (C) and ONL/OPL/INL region (D) depictured as 3D reconstruction (Edn2/merge/3D surface rendering). C. Edn2 signals (red, arrow) partly overlapped with GFAP (green) - positive astrocytes. D. Edn2 punctae (red, arrow) overlapped to some extent with GS (purple) -positive resting (control animal, arrow) and GFAP (green)/ GS (purple) -positive reactive (VPP animal, arrow) Müller cells. E. In situ hybridization for Ednrb and GFAP / GS immunofluorescence colabeling in the retinae of three-month-old animals. Nuclei were DAPI-stained (blue). Ednrb (red, arrowheads) signals were detectable in the ONL and INL with some sparse signals in the GCL of control and VPP animals. In the VPP retina, the Müller cells were GFAP (green)/ GS (purple) - positive, indicating their reactivity. Ednrb signals (red) were visible in the INL and ONL that did not overlap with GFAP/ GS, indicating its further expression in neuronal cells. F. Boxplots showing the extracted Ednrb expression data from the RNAseq as normalized counts for control and VPP genotypes. Control $n=6$; VPP $n=5$; padj $=0.0074$. G. and $\mathrm{H}$. Higher magnification of the GCL $(G)$ and ONL/OPL/INL region $(\mathrm{H})$ depictured as 3D reconstruction (Ednrb/merge/3D surface rendering). G. Ednrb signals (red, arrow) partly overlapped with GFAP (green) - positive astrocytes. H. Ednrb punctae (red, arrow) overlapped to some extent with GS (purple) -positive resting (control animal, arrow) and GFAP (green)/ GS (purple) -positive reactive (VPP animal, arrow) Müller cells. Edn2 = endothelin2; Ednrb = endothelin receptor type B; GCL = ganglion cell layer; INL = inner nuclear layer; $\mathrm{OPL}=$ outer plexiform layer; $\mathrm{ONL}=$ outer nuclear layer; $\mathrm{GFAP}$ = glial fibrillary acidic protein; $G S=$ glutamine synthetase; $A B / p r$. co. = antibody $/$ probe control. 

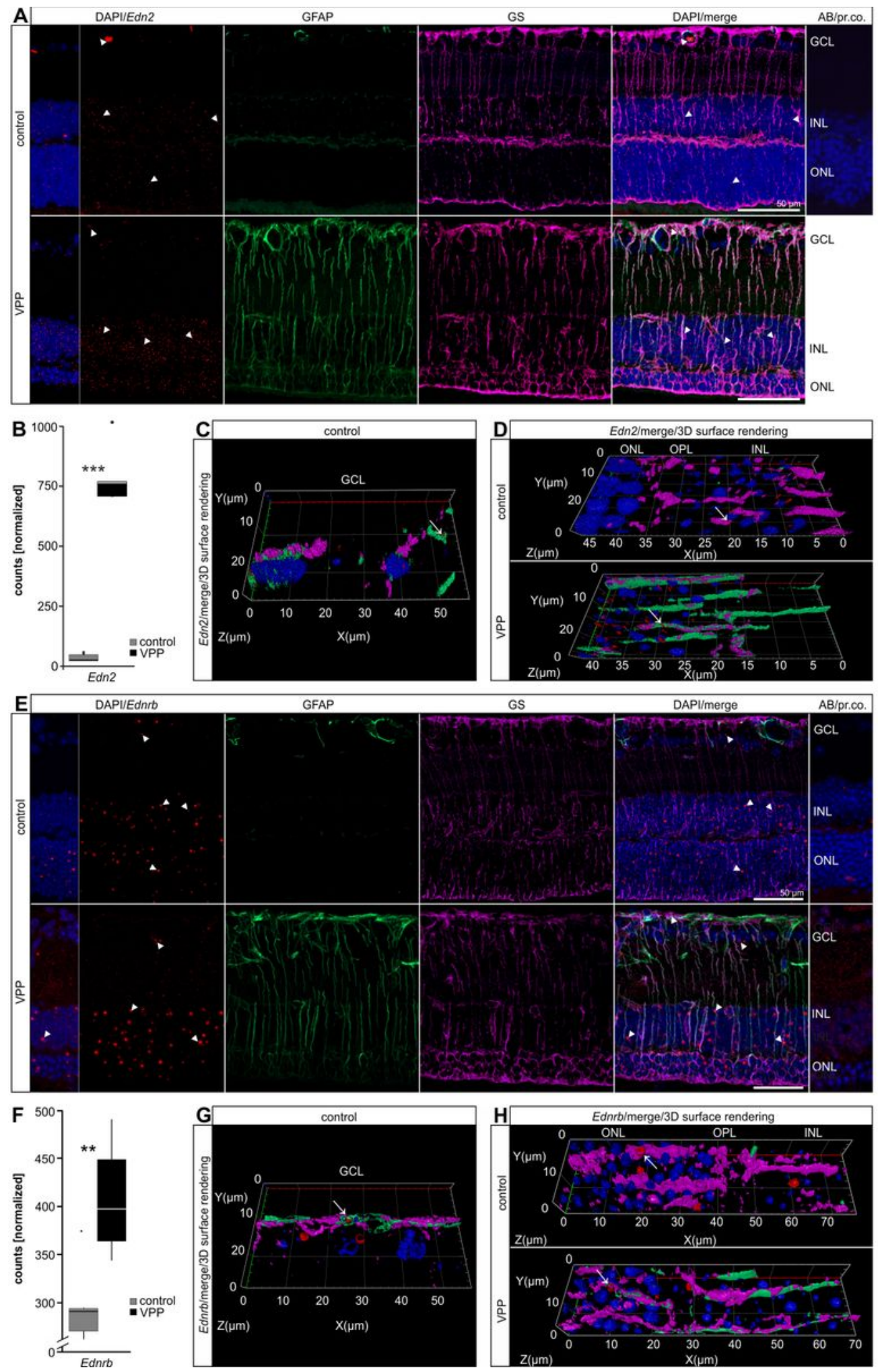

Figure 5

Upregulation of endothelin signaling in VPP mice A. In situ hybridization for Edn2 (red) and GFAP (green) /GS (purple) immunofluorescence co-labeling in the retinae of three-month-old animals. Nuclei were DAPIstained (blue). Edn2 (red, arrowheads) signals were detectable in the ONL and INL with some sparse signals in the GCL. In the VPP retina, the number of the Edn2 signals (red, arrowheads) was increased in the ONL and INL and the Müller cells were GFAP (green) /GS (purple) -positive, indicating their reactivity. 
Edn2 signals (red) were visible in the INL and ONL that did not overlap with GFAP/ GS, indicating its additional expression in neuronal cells. B. Boxplots showing the extracted Edn2 expression data from the RNAseq as normalized counts for control and VPP genotypes. Control $n=6$; VPP $n=5 ;$ padj $=8.68 \cdot 10-87$. C. and D. Higher magnification of the GCL (C) and ONL/OPL/INL region (D) depictured as 3D reconstruction (Edn2/merge/3D surface rendering). C. Edn2 signals (red, arrow) partly overlapped with GFAP (green) - positive astrocytes. D. Edn2 punctae (red, arrow) overlapped to some extent with GS (purple) -positive resting (control animal, arrow) and GFAP (green)/ GS (purple) -positive reactive (VPP animal, arrow) Müller cells. E. In situ hybridization for Ednrb and GFAP / GS immunofluorescence colabeling in the retinae of three-month-old animals. Nuclei were DAPI-stained (blue). Ednrb (red, arrowheads) signals were detectable in the ONL and INL with some sparse signals in the GCL of control and VPP animals. In the VPP retina, the Müller cells were GFAP (green)/ GS (purple) - positive, indicating their reactivity. Ednrb signals (red) were visible in the INL and ONL that did not overlap with GFAP/ GS, indicating its further expression in neuronal cells. F. Boxplots showing the extracted Ednrb expression data from the RNAseq as normalized counts for control and VPP genotypes. Control $n=6$; VPP $n=5$; padj $=0.0074$. G. and $\mathrm{H}$. Higher magnification of the GCL $(G)$ and ONL/OPL/INL region $(\mathrm{H})$ depictured as 3D reconstruction (Ednrb/merge/3D surface rendering). G. Ednrb signals (red, arrow) partly overlapped with GFAP (green) - positive astrocytes. H. Ednrb punctae (red, arrow) overlapped to some extent with GS (purple) -positive resting (control animal, arrow) and GFAP (green)/ GS (purple) -positive reactive (VPP animal, arrow) Müller cells. Edn2 = endothelin2; Ednrb = endothelin receptor type B; GCL = ganglion cell layer; INL = inner nuclear layer; $\mathrm{OPL}=$ outer plexiform layer; $\mathrm{ONL}=$ outer nuclear layer; $\mathrm{GFAP}$ = glial fibrillary acidic protein; $G S=$ glutamine synthetase; $A B / p r$. co. = antibody $/$ probe control. 

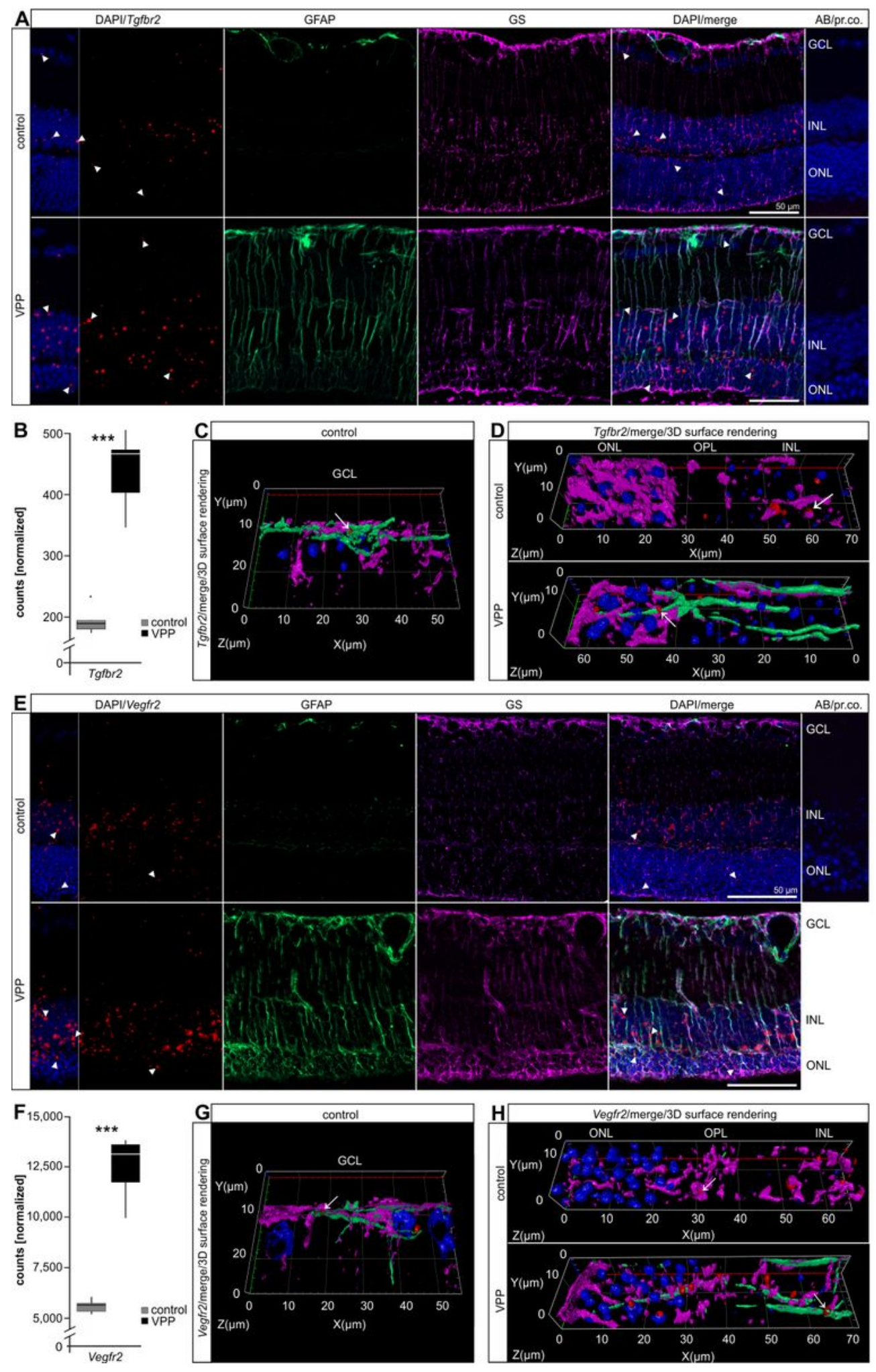

Figure 6

Upregulation of TGF $\beta$ - and VEGF-signaling in VPP mice A. In situ hybridization for Tgfbr2 (red) and GFAP (green) /GS (purple) immunofluorescence co-labeling in the retinae of three-month-old animals. Nuclei were DAPI-stained (blue). Tgfbr2 (red, arrowheads) signals were detectable in the ONL and INL with some isolated punctae in the GCL. In the VPP retina, number of Tgfbr2 signals (red, arrowheads) in the ONL and INL was increased and the Müller cells were GFAP (green)/GS (purple) -positive, indicating their reactivity. 
Additionally, Tgfbr2 signals were visible in the INL and ONL that did not overlap with GFAP/GS, indicating its further expression in the neuronal cells. B. Boxplots showing the extracted Tgfbr2 expression data from the RNAseq as normalized counts for control and VPP genotypes. Control $n=6 ;$ VPP $n=5 ;$ padj $=$ 2.18-10-23. C. and D. Higher magnification of the GCL (C) and ONL/OPL/INL region (D) depictured as 3D reconstruction (Tgfbr2/merge/3D surface rendering). C. Tgfbr2 signals (red, arrow) showed only scattered co-labeling with GFAP (green) -positive astrocytes. D. Tgfbr2 punctae (red, arrow) partly associated with GS (purple) -positive resting (control animal, arrow) and GFAP (green)/ GS (purple) -positive reactive (VPP animal, arrow) Müller cells. E. In situ hybridization for Vegfr2/Kdr (red) and GFAP (green) /GS (purple) immunofluorescence co-labeling in the retinae of three-month-old animals. Nuclei were DAPI-stained (blue). Vegfr2 (red, arrowheads) signals were visible in the ONL and INL with some isolated signals in the GCL. The number of Vegfr2 signals (red, arrowheads) was increased in the VPP retina and the Müller cells were GFAP (green)/GS (purple) -positive, indicating their reactivity. Vegfr2 signals were visible in the INL and ONL that did not overlap with GFA/GS indicating its expression in the neuronal cells. F. Boxplots showing the extracted Vegfr2/Kdr expression data from the RNAseq as normalized counts for control and VPP genotypes. Control $n=6$; VPP $n=5 ;$ padj $=1.40 \cdot 10-41 . \mathrm{G}$. and $\mathrm{H}$. Higher magnification of the GCL (G) and ONL/OPL/INL region $(\mathrm{H})$ depictured as 3D reconstruction (Vegfr2/merge/3D surface rendering). $\mathrm{G}$. Vegfr2 signals (arrow) showed some co-labeling with GFAP (green) -positive astrocytes. H. Vegfr2 signals partly overlapped with GS (purple) -positive resting (control animal, arrow) and GFAP (green)/ GS (purple) -positive reactive (VPP animal, arrow) Müller cells. Tgfbr2 = transforming growth factor beta receptor type 2; Vegfr2 = vascular endothelial growth factor receptor 2; $\mathrm{GCL}=$ ganglion cell layer; $\mathrm{INL}=$ inner nuclear layer; $\mathrm{OPL}=$ outer plexiform layer; $\mathrm{ONL}=$ outer nuclear layer; $\mathrm{AB} / \mathrm{pr}$. co. = antibody $/$ probe control. 

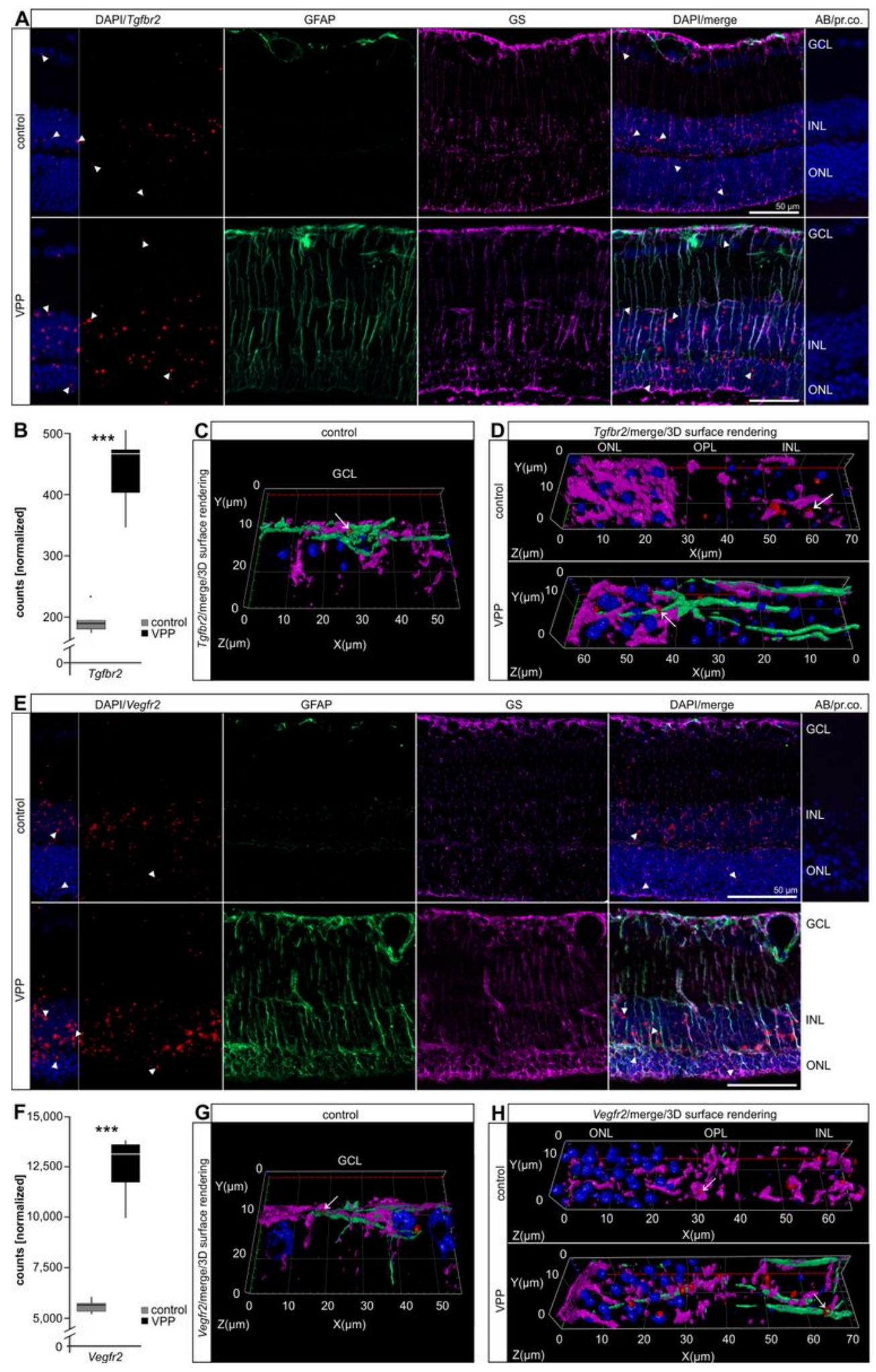

Figure 6

Upregulation of TGF $\beta$ - and VEGF-signaling in VPP mice A. In situ hybridization for Tgfbr2 (red) and GFAP (green) /GS (purple) immunofluorescence co-labeling in the retinae of three-month-old animals. Nuclei were DAPI-stained (blue). Tgfbr2 (red, arrowheads) signals were detectable in the ONL and INL with some isolated punctae in the GCL. In the VPP retina, number of Tgfbr2 signals (red, arrowheads) in the ONL and INL was increased and the Müller cells were GFAP (green)/GS (purple) -positive, indicating their reactivity. 
Additionally, Tgfbr2 signals were visible in the INL and ONL that did not overlap with GFAP/GS, indicating its further expression in the neuronal cells. B. Boxplots showing the extracted Tgfbr2 expression data from the RNAseq as normalized counts for control and VPP genotypes. Control $n=6 ;$ VPP $n=5 ;$ padj $=$ 2.18-10-23. C. and D. Higher magnification of the GCL (C) and ONL/OPL/INL region (D) depictured as 3D reconstruction (Tgfbr2/merge/3D surface rendering). C. Tgfbr2 signals (red, arrow) showed only scattered co-labeling with GFAP (green) -positive astrocytes. D. Tgfbr2 punctae (red, arrow) partly associated with GS (purple) -positive resting (control animal, arrow) and GFAP (green)/ GS (purple) -positive reactive (VPP animal, arrow) Müller cells. E. In situ hybridization for Vegfr2/Kdr (red) and GFAP (green) /GS (purple) immunofluorescence co-labeling in the retinae of three-month-old animals. Nuclei were DAPI-stained (blue). Vegfr2 (red, arrowheads) signals were visible in the ONL and INL with some isolated signals in the GCL. The number of Vegfr2 signals (red, arrowheads) was increased in the VPP retina and the Müller cells were GFAP (green)/GS (purple) -positive, indicating their reactivity. Vegfr2 signals were visible in the INL and ONL that did not overlap with GFA/GS indicating its expression in the neuronal cells. F. Boxplots showing the extracted Vegfr2/Kdr expression data from the RNAseq as normalized counts for control and VPP genotypes. Control $n=6$; VPP $n=5 ;$ padj $=1.40 \cdot 10-41 . \mathrm{G}$. and $\mathrm{H}$. Higher magnification of the GCL (G) and ONL/OPL/INL region $(\mathrm{H})$ depictured as 3D reconstruction (Vegfr2/merge/3D surface rendering). $\mathrm{G}$.

Vegfr2 signals (arrow) showed some co-labeling with GFAP (green) -positive astrocytes. H. Vegfr2 signals partly overlapped with GS (purple) -positive resting (control animal, arrow) and GFAP (green)/ GS (purple) -positive reactive (VPP animal, arrow) Müller cells. Tgfbr2 = transforming growth factor beta receptor type 2; Vegfr2 = vascular endothelial growth factor receptor 2; $\mathrm{GCL}=$ ganglion cell layer; $\mathrm{INL}=$ inner nuclear layer; $\mathrm{OPL}=$ outer plexiform layer; $\mathrm{ONL}=$ outer nuclear layer; $\mathrm{AB} / \mathrm{pr}$. co. = antibody $/$ probe control.

\section{Supplementary Files}

This is a list of supplementary files associated with this preprint. Click to download.

- Figsuppl1networks01.tif

- Figsuppl1networks01.tif

- Figsuppl2heatmaps01.tif

- Figsuppl2heatmaps01.tif

- Figsuppl3pathways01.tif

- Figsuppl3pathways01.tif

- Figsuppl4ortho.tif

- Figsuppl4ortho.tif 